\title{
Hypo- or hyperactivity of zebrafish embryos provoked by neuroactive substances: a review on how experimental parameters impact the predictability of behavior changes
}

\author{
Afolarin Ogungbemi ${ }^{1}{ }^{, 2^{*}} \mathbb{D}$, David Leuthold ${ }^{1}$, Stefan Scholz ${ }^{1}$ and Eberhard Küster ${ }^{1}$
}

\begin{abstract}
Tests with zebrafish embryos have gained wide acceptance as an alternative test model for drug development and toxicity testing. In particular, the behavioral response of the zebrafish embryo is currently seen as a useful endpoint to diagnose neuroactive substances. Consequently, several behavioral test methods have been developed addressing various behavioral endpoints such as spontaneous tail coiling (STC), photomotor response (PMR), locomotor response (LMR) and alternating light/dark-induced locomotor response (LMR-L/D). Although these methods are distinct in their application, most of their protocols differ quite strongly in the use of experimental parameters and this is usually driven by different research questions. However, if a single mode of action is to be diagnosed, then varying experimental parameters may cause incoherent behavioral responses (hypo- or hyperactivity) of zebrafish during toxicity assessment. This could lead to inconclusiveness of behavioral test results for use within a prospective and diagnostic risk assessment framework. To investigate the influence of these parameters, we conducted a review of existing behavioral assays to address the following two questions: (1) To what extent do varying experimental parameters influence observed effects in published behavioral test methods? (2) Is the observed behavior change (hypo- or hyperactivity) of zebrafish embryos consistent with the expected mode of action of a chemical? We compiled a set of 18 substances which are anticipated to be neuroactive. We found that behavioral changes are not only affected by chemicals but also variation in the use of experimental parameters across studies seems to have a high impact on the outcome and thus comparability between studies. Four parameters, i.e., exposure concentration, exposure duration, endpoint parameter and developmental stage were the most influential parameters. Varying combinations of these parameters caused a non-reproducible outcome for the hyperactivity expected for the organophosphates; chlorpyrifos and diazinon. We highlighted that the STC test shows a higher capacity to predict the hyperactivity of organophosphates, while PMR and LMR-L/D were more suitable to predict the hypoactivity expected for anticonvulsants. We provide a list of recommendations which, when implemented, may help to exclude the risk of bias due to experimental parameters if similar goals are desired.
\end{abstract}

Keywords: Neurotoxicity, Activity, Hypoactivity, Anticonvulsants, Acetylcholinesterase inhibitors, Pesticides, Pharmaceuticals, Behavioral ecotoxicology, Risk assessment

\footnotetext{
*Correspondence: afolarin.ogungbemi@ufz.de

1 Dept. Bioanalytical Ecotoxicology, Helmholtz Centre for Environmental

Research-UFZ, Permoserstr. 15, 04318 Leipzig, Germany

Full list of author information is available at the end of the article
} 


\section{Background}

Many chemical substances released into the environment exhibit neuroactive properties and may have negative consequences on human and environmental health [67]. In fact, most of these substances are designed to interact with the nervous system. For example, insecticides target the nervous system of invertebrates, while some pharmaceuticals are designed to treat neurodegenerative diseases in humans [26, 107]. Although chemical monitoring techniques are commonly used, a holistic approach for risk assessment requires appropriate effect-based tools to detect neurotoxic effects [11]. Busch et al. [15] showed that neuroactive substances represent the largest group $(13 \%)$ of chemicals with known mode of action (MoA) detected in European rivers. These neuroactive substances co-exist in the environment with other chemicals [67] and substance-receptor relationships could be useful to identify these neuroactive substances within a complex mixture. Typically, neuroactive substances target specific parts of the nervous system such as acetylcholinesterases (AChE), nicotine receptors (nAChr), gamma-aminobutyric acid receptors (GABA) and sodium channel receptors. Despite the widespread occurrence of neuroactive chemicals in the environment and their ability to disrupt the nervous system, standardized methods for assessing the risks of these substances are lacking [66]. Hence, we conducted a review of the commonly used behavior test methods in zebrafish embryo which are used to assess neurotoxicity. We collated behavior testing of 18 substances from different studies. This review evaluates thecomparability of the experimental parameters used in these studies as a means to optimize them for assessing and detecting neuroactive substances within a prospective and diagnostic risk assessment framework.

\section{Importance of zebrafish embryo behavior}

Testing of the adverse effects of chemicals to humans and the environment relies at present to a large extent on animal models such as rodents and adult fish [72]. It is, however, known that the exposure of animals to chemicals may inflict pain and distress. Hence, there is an increased need to develop alternatives to animal tests because of ethical reasons, as well as to reduce time and cost of these tests $[12,29]$. Consequently, the use of animals in toxicity testing has been highly discouraged in favor of promoting the $3 \mathrm{R}$ principle: reduction, refinement and replacement $[33,96]$. In turn, the use of cell lines is encouraged as an alternative due to their ability to identify mechanisms underlying toxic effects [100]. However, the inability of cell lines to integrate the interaction of various tissues within a multicellular system is a major disadvantage [102]. Alternatively, zebrafish embryos proved to be a promising model due to its capacity to predict fish and rodent toxicity [47, 103]. Further, the behavior of zebrafish embryos can be used to distinguish between different neurotoxic MoA such as beta-adrenergic receptor agonists, dopamine agonists and adenosine receptor antagonists [60].

In fish, functional interference with the cardiovascular and nervous system, particularly demonstrated for AChE inhibition, leads to the respiratory failure syndrome resulting in enhanced mortality due to oxygen limitations [97]. In contrast, fish embryos appear to lack the respiratory failure syndrome because oxygen in embryos is mainly supplied via skin diffusion $[59,95]$. As a result, embryos only show a weak mortality for neurotoxic substances. However, it has been shown that some neuroactive substances exhibit effects on behavior at concentrations well below the lethal range [59]. Hence, the observation of behavior changes at sublethal concentration ranges in embryos may provide an indicator for neuroactivity and/or could be used to infer adverse effects. Therefore, the aim of this review is to identify experimental parameters for behavior assays that could support the unbiased diagnosis of different neuroactive mode of actions.

\section{Types of behavioral tests considered in this review}

The potential to identify interactions of chemicals with the nervous system using behavioral assays in zebrafish has been recognized and several behavioral test methods have been developed. In this review, we focus on the most commonly utilized tests and their endpoints including spontaneous tail coiling (STC), photomotor response (PMR), locomotor response (LMR) and alternating light- and dark-induced locomotor response (LMR-L/D) (Table 1).

Spontaneous tail coiling (STC) is the first motor activity generated by the developing neural network which occurs as a result of the innervation of the muscle [58]. This event is assumed to be important for the hatching of the embryo from its chorion but evidence for a role of STC for hatching has not yet been provided $[58,98]$. Frequency changes of the STC have been used as a tool to detect the effects of neuroactive chemicals in developing embryo [118, 121].

The photomotor response (PMR) is an embryonic movement induced by a high-intensity light stimulus (wavelength between 300 and $700 \mathrm{~nm}$ ). This response is independent from light perception by the eyes and mediated through photoreceptors in the developing hindbrain $[60,89]$. The PMR can be divided into four broad phases, a pre-stimulus background phase, latency phase, excitation phase and refractory phase. The visualization of 
Table 1 Characteristics of different behavioral tests

\begin{tabular}{|c|c|c|c|c|}
\hline & $\begin{array}{l}\text { Spontaneous tail } \\
\text { coiling test (STC) }\end{array}$ & Photomotor response test (PMR) & $\begin{array}{l}\text { Locomotor } \\
\text { response test } \\
\text { (LMR) }\end{array}$ & $\begin{array}{l}\text { Alternating light- and dark-induced } \\
\text { locomotor response test (LMR-L/D) }\end{array}$ \\
\hline Applied stimuli & Non & High-intensity light & Non & Alternating light/dark \\
\hline Endpoint & $\begin{array}{l}\text { Number or } \\
\text { frequency of tail } \\
\text { coilings }\end{array}$ & Movement activity or motion index & $\begin{array}{l}\text { Swimming dis- } \\
\text { tance, duration, } \\
\text { and speed }\end{array}$ & Swimming distance, duration, and speed \\
\hline Exposure duration & 0-28 hpf & $0-42 \mathrm{hpf}$ & $0-120 \mathrm{hpf}$ & 0-120 hpf \\
\hline $\begin{array}{l}\text { Age of embryo at time } \\
\text { of measurement }\end{array}$ & 19-28 hpf & $28-42 \mathrm{hpf}$ & 72-120 hpf & $72-120 \mathrm{hpf}$ \\
\hline
\end{tabular}

these PMR phases has been used for chemical classification and drug screening [60]. Both STC and PMR represent endpoints measured in pre-hatching embryo stages (19-42 hpf) of zebrafish.

The locomotor response can either be spontaneous (LMR) or induced with alternating light/dark periods (LMR-L/D) and these can be measured in the post-hatching embryo stages ( $>48 \mathrm{hpf}$ ) of zebrafish. In LMR-L/D, zebrafish embryos exhibit weak movement when illuminated by light but exhibit an increase in activity when switched from light to dark [52, 106]. Therefore, lightdark cycles are applied to monitor this behavior. LMR is estimated by recording various swimming activity endpoints such as swimming time, swimming distance, swimming speed (calculated from distance and time) and swimming angle, while LMR-L/D measures the stated parameters under alternating light/dark cycles. The LMR assessment is similar to behavioral monitoring studies that use adult fish, e.g., for online (bio-) monitoring of waste- or surface water, also known as "fish toximeters". In these toximeters, for example, alteration of swimming activity is often used as an indicator of potential adverse effects due to chemical exposure [3].

\section{Influence of experimental parameters}

Although these behavioral test methods, particularly LMR and PMR, are widely used and have been applied in large drug and chemical screens $[14,89]$, they differ largely in experimental parameters between different labs such as exposure duration, selection of endpoint parameter, etc. Several authors have studied the influence of experimental parameters on zebrafish embryo behavior. For example, distance moved by zebrafish embryo varies with age of embryos and size of exposure vessel [27, 82]; light/dark response is usually affected by duration of cycles and the number of repeats [76]; and the observed effect is highly dependent on the exposure concentration or endpoint selected [27, 50]. Legradi et al. [66] reviewed the literature to compare behavioral test methods. They found that for LMR assessment, experimental parameters such as duration of behavior assay and developmental stage of embryos varied significantly among studies. It is still largely unknown how this variability influences the outcome of behavioral studies. The need to harmonize and report experimental parameters has previously been discussed for zebrafish embryotoxicity test [8] and in vitro neurotoxicity testing [23]. However, such critical discussion and analysis for neurotoxicity testing using zebrafish embryo behavior are still lacking.

\section{The use of hypo- and hyperactivity as a predictor}

In some studies, the differentiation between hypo- and hyperactivity has been suggested as a potential indicator for neuroactivity MoA [31, 38, 60]. With respect to embryonic behavior of unexposed embryos, hypoactivity refers to a decrease in the spontaneous or induced movement of embryos, while hyperactivity refers to the opposite. In this study, we define neurotoxicity as an interaction of a substance with the nervous system primarily leading to a structural change (e.g., axonal deformation or inhibition of neurite outgrowth), while neuroactivity is a functional interaction with specific nervous receptors leading to a change without necessarily being accompanied by structural changes (e.g., alteration of neurotransmission or blockage of nervous receptor). Nonetheless, neuroactivity may lead secondarily also to structural and morphological changes over a longer duration or higher concentration of chemical exposure. It is assumed that neuroactive substances are able to modulate nervous receptors leading to hypo- or hyperactivity behavior [114]. For example, Vliet et al. [118] used the STC response as a metric to screen a library of 1280 pharmacologically active compounds for neuroactivity. Reif et al. [89] used the hypo- or hyperactivity observed in the different phases of the PMR to characterize a suite of 1060 chemicals; and Bugel and Tanguay [14] were able to negate a GABA mode of action for a suite of 24 flavonoids in the LMR based on the induction of hypo- or hyperactivity. 
The rationale behind the above-mentioned screening studies was that substances with the same or similar mode of action would only induce either hypo- or hyperactivity. However, it is also possible that both hypo- and hyperactivity (biphasic activity) may be induced by the same substance depending on the concentration level or duration of exposure. For example, chlorpyrifos-oxon and aldicarb-sulfoxide stimulate nerve cells by inhibiting acetylcholinesterase, thereby inducing hyperactivity and increased heartbeat of embryos, respectively [62, 120]. At higher concentrations, the over-excitation of the cholinergic system may result in paralysis caused by seizures and thus leading to hypoactivity [111]. Alternatively, abamectin induces hypoactivity due to its inhibitory action when it activates the GABA-gated chloride channel [87]. Such distinct characteristics of neuroactive substances suggest that the hypo- or hyperactivity of zebrafish embryos may be used to identify MoA when experimental parameters are adequately controlled [6].

\section{Aims and approach of this review}

We aimed to investigate the influence of experimental parameters on the hypo- or hyperactivity response of zebrafish embryos by reviewing existing literature. We first created a collection of pharmaceutical and pesticide substances with known MoA, for which sufficient information on effect concentrations and experimental parameters were available, to address the following questions:

1. Which experimental parameter(s) mostly influence the observed effects in the four above-mentioned behavioral test methods and is it possible to rank these parameters?

2. How often is the observed hypo- or hyperactivity of zebrafish embryos in literature consistent with the expected mode of action of a chemical substance?

These questions are based on the hypo- or hyperactivity hypothesis, i.e., whether the behavioral response (hypo- or hyperactivity in moved distance, tail coiling or else) of zebrafish embryos predicts the mode of action of a neuroactive chemical and vice versa. This review provides information that will support the selection of a combination of appropriate behavioral tests within a prospective risk assessment framework. We also present critical evaluations on how to use hypo- and hyperactivity detection as a tool to improve the identification of neuroactive mode of action in a complex mixture within a diagnostic risk assessment framework.

\section{Literature selection}

Literature search was mainly undertaken by searching the "web of science" database (http://www.webof knowledge.com) and the search results were filtered in a KNIME $^{\circledR}$ (http://www.knime.com) workflow. A few additional papers were selected by cross-referencing of citations. Figure 1 shows a summary of the literature selection procedure.

1. Keyword search on "web of science" database: The following keywords combinations were searched for representation in either title, abstract or keywords: "zebrafish embryo" OR "zebrafish larvae" OR zebrafish) AND ("throughput" or locomotor OR LMR OR "spontaneous activity" OR STC OR Photomotor OR PMR) AND (behav* OR hyperactivity OR hypoactivity OR neurotoxicity OR movement). Only studies published between the years 2000 and 2018 were retrieved. The search was conducted on 17 th August 2018. The search was repeated on 29th July 2019 to include additional hits of chemicals that were already included in the analysis.

2. Apply exclusion criteria by screening the abstract of the study hits: The following exclusion criteria were

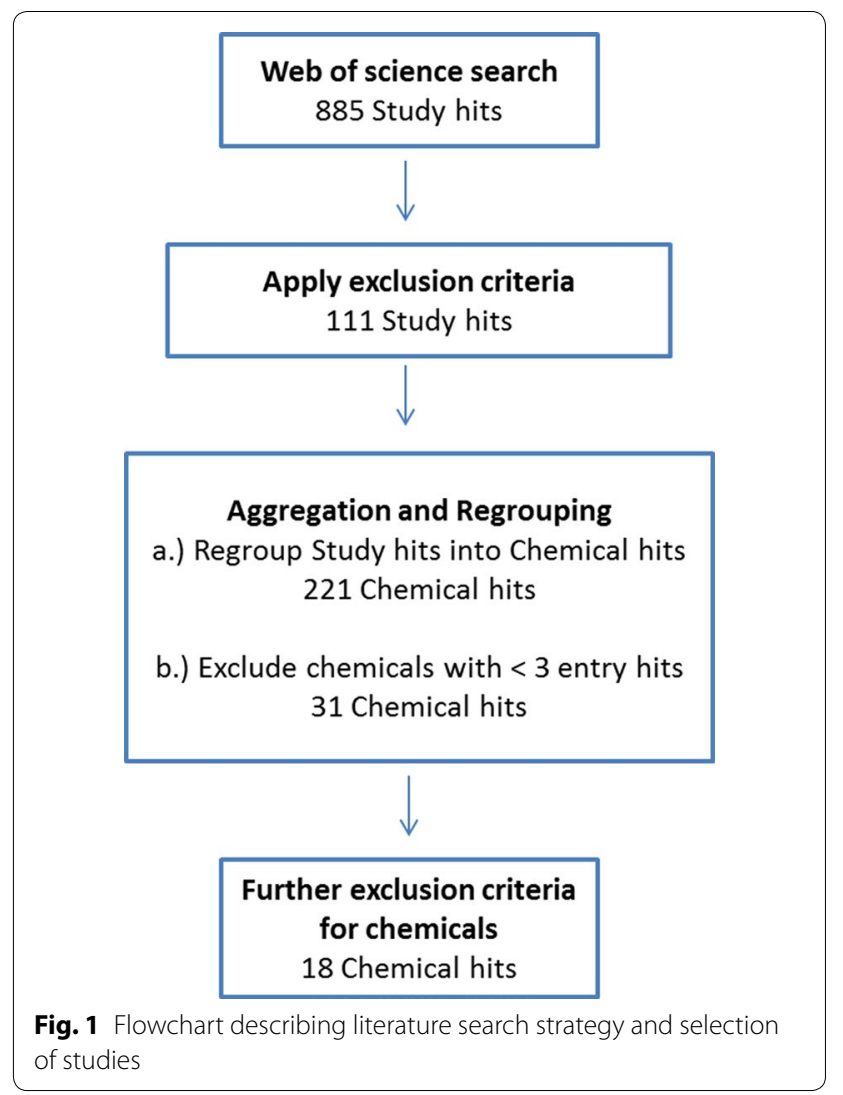


applied to reduce the variability between studies and to increase the quality of data collection.

a. Organism: Not zebrafish, or a mutant zebrafish strain was used.

b. Age: Juvenile or adult stages were tested.

c. Method: Other assays than STC, PMR, LMR and LMR-L/D were used.

d. Chemical: Only a mixture, an inorganic compound or a natural, undefined product was used.

e. Exposure: Oral exposure

f. Effect: No report or indication of hypo- or hyperactivity.

3. Regrouping of study hits into chemical hits:

a. A KNIME ${ }^{\circledR}$ workflow was used to aggregate and regroup the study hits according to the test chemical. The $\mathrm{KNIME}^{\circledR}$ workflow is shown in Additional file 1: Figure S1.

b. The KNIME ${ }^{\circledR}$ workflow was also used to exclude chemicals with less than 3 entry hits

4. A further exclusion criterion was applied to the chemical hits to increase the quality of the collected data; i.e., chemicals with unknown or unclear mode of action were excluded.

\section{Data collection and analysis}

After selecting the appropriate studies based on the criteria above, we proceeded to analyze data reported in these studies. Data collected included: chemicaltype, mode of action, effect concentration (for hyper- and hypoactivity), exposure duration, analysis duration, exposure well size, developmental stage at exposure, zebrafish strain, etc. The full raw data collected are available in Additional file 2: Excel sheet 3.

\section{Results and discussion}

In the first part of the results, we address the questionwhich experimental parameters are mostly influencing toxicity outcome of behavioral tests and we considered parameters related to exposure design and effect measurement for this analysis.

In the second part of the results, we address the question-how often the observed hypo- or hyperactivity of zebrafish embryos after exposure to a chemical is consistent with the expected mode of action of this chemical. We compared observed and expected activities at three levels: a. Individual chemical comparison, i.e., individual chemicals were selected and the found literature results were compared on the level of observed behavior effect;

b. Chemical class comparison, i.e., do organophosphates, for example, always show the same activity change in all behavior assays, even if test parameters were different;

c. Behavioral method comparison, i.e., whether different behavioral test methods or contrasting experimental parameters give similar results?

For the ranking of the influence of each parameter, we analyzed the percentage concordance of hyperactivity between expected and observed activity for each behavioral test method. This was estimated by dividing the number of studies in which hypo- or hyperactivity was observed by the total number of studies for a certain chemical expected to cause hypo- or hyperactivity. Any observed non-concordance was attributed to experimental parameters as a risk of bias factor.

\section{Influence of experimental parameters on behavior analysis}

Thirteen experimental parameters were identified in the literature survey with potential impact on the outcome of different behavior tests. The parameters were subgrouped into biological (or intrinsic) and technical (or extrinsic) experimental factors. Developmental stage, zebrafish strain, malformations, rearing conditions, time of day for behavior analysis and the selected endpoints were considered as biological factors. Exposure duration, exposure concentration, duration of behavior analysis, exposure well size, material used for exposure vessel, light conditions and solvent concentration were considered as technical factors. Table 2 shows the potential influence of the 13 experimental parameters which are more precisely explained in the text below.

\section{Biological (intrinsic) factors Developmental stage}

Padilla et al. [82] investigated the influence of development stage on distance moved in alternating light and dark periods. They compared the behavior of 4-, 5- and 6-day-old non-exposed embryos/larvae in the light period and found that older embryos had increased movement, indicated by the distance moved, than younger ones. On the other hand, the influence of age in the dark period was not obvious except for differences in the pattern of movement. The observed influence of age on behavior in the light period may be due to differences in retinal maturation as the retina ganglia cells of younger larvae may not respond to light as much as older larvae 


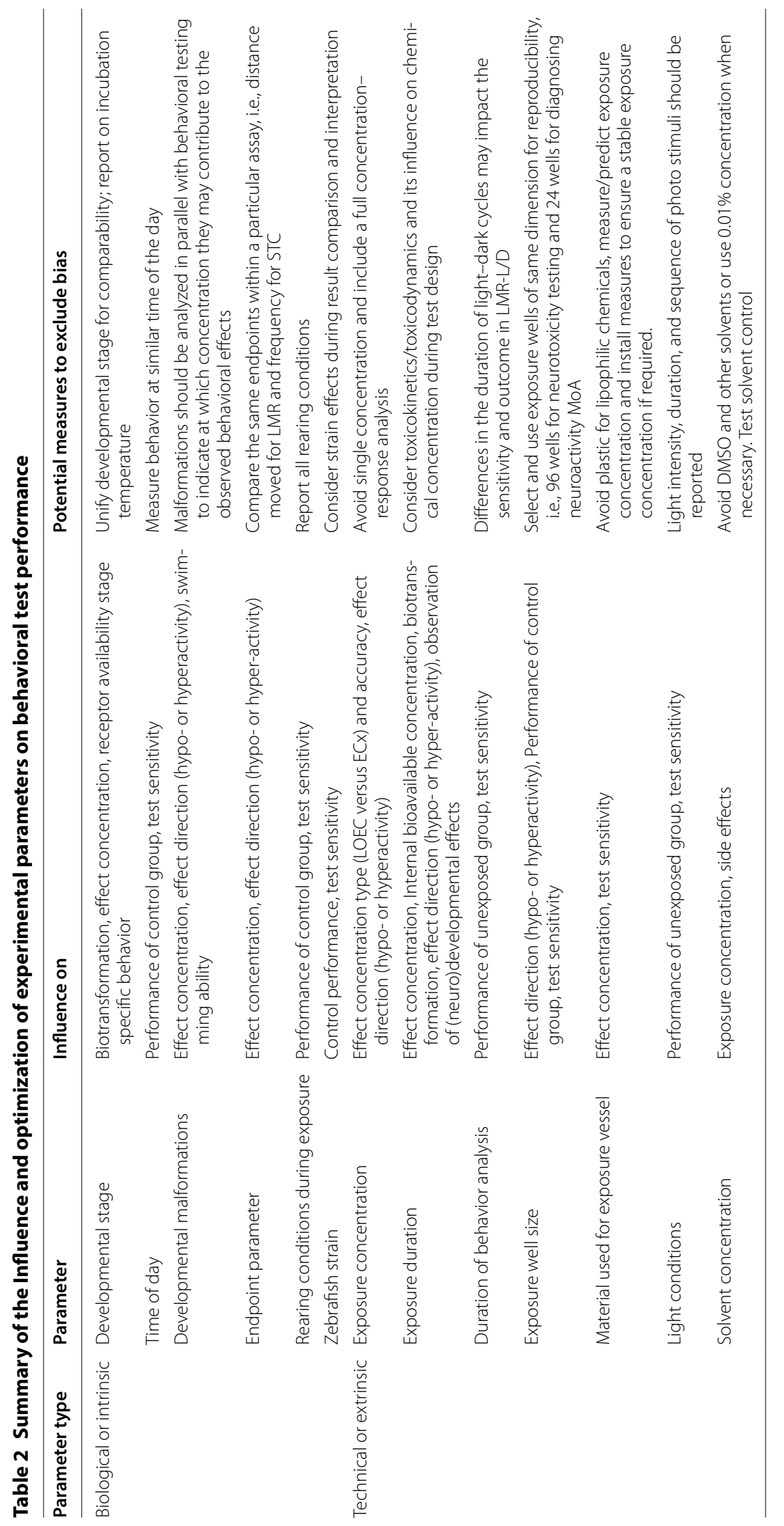


[82]. These findings are corroborated by De Esch et al. [27], who compared 5-, 6- and 7-day-old embryos/larvae and Leuthold et al. [68] who compared 4- and 5-day-old embryos. Fraser et al. [38] reported increased movement for older embryos $(120>100>96 \mathrm{hpf})$ when raised in constant darkness. In contrast, Ingebretson and Masino [50], while considering total distance moved in constant light, reported that 7-day-old larvae moved less than 4-day-old embryos. However, the impact of age on the distance moved was only observed when embryos were analysed in deep but not in shallow wells. The developmental stage may not only impact the control behavior but also can impact the observed responses to exposure of chemicals that require biotransformation such as organophosphates [61]. Furthermore, some neurotransmitters such as histamine and 3-methoxytyramine may not be present in early developmental stages and this could lead to differences in observed effects due to target availability $[94,116]$. Principally, differences in the uptake kinetics of chemicals may also impact the behavior at specific developmental stages. However, so far, there is weak evidence for considerable uptake differences [78]. Nevertheless, for pre-hatched stages and high-molecularweight compounds, the chorion may represent a barrier $[84,102]$. These findings indicate that the developmental stage at which the analysis is conducted may be influential. Relative effects by chemicals may not necessarily be disturbed if they are measured at the same stage.

\section{Time of the day for analysis}

Kristofco et al. [61] investigated the influence of daytiming on behavior analysis in alternating light/dark test (LMR-L/D). They found that activity of embryos and larvae measured daily (between 4 and $10 \mathrm{dpf}$ ) were significantly lower in the early hours of the day at 9 and 10 a.m. and more variable during these periods, while activity was higher and less variable in the afternoon. In contrast, MacPhail et al. [76] measured locomotion and reported higher movement for $6 \mathrm{dpf}$ larvae at 10 and 11 a.m. in the morning, while lower movement was recorded in the afternoon. The two studies used the same 14/10 light/ dark cycle. MacPhail et al. [76] recorded behavior only for the dark period. Both authors concluded that activity was less variable when measured in the afternoon. In contrast to both studies, Fitzgerald et al. [37] reported that time of day did not change the activity of 5, 6 and $7 \mathrm{dpf}$ larvae in the dark period of LMR-L/D measured at 9 a.m and 2 p.m., and activity was more variable in the afternoon rather than in the morning. However, a different temperature $\left(26^{\circ} \mathrm{C}\right.$ versus $28^{\circ} \mathrm{C}$ of the other studies) was used as rearing temperature and the controversial finding may have been caused by differences in growth and developmental stage. Furthermore, the influence of day-time may rather relate to the time after the onset of light, but details on light cycles are missing in some articles. These results suggest that diurnal rhythm of fish embryos and the impact on responses may have to be controlled by measuring behavior at similar time of the day, and that impacts of growth and developmental as potential confounding factors should be considered.

\section{Developmental malformations}

Padilla et al. [82] reported that malformed zebrafish in control solution showed hypoactivity in both light and dark periods, while healthy control animals remained unaffected in behavior. Hence, reduced movement of activity may represent a secondary effect following malformations when embryos are exposed to chemicals. Consequently, malformations should be analyzed in parallel and concentration-response data for phenotypic effects and behavior should be compared to identify potential secondary effects. By comparing the effect concentration for behavior and malformation, the specificity of the behavioral effects could be assessed. However, subtle alterations at sub-organism level (e.g., muscle structure) not easily detectable by microscopical observations may also impact the behavior.

\section{Endpoint parameter}

De Esch et al. [27] compared three different endpoints in LMR-L/D, namely distance moved, duration of movement and swimming velocity (calculated from distance and time), among three different developmental stages. They found that results for swimming velocity were negatively correlated with the other endpoints, i.e., high velocity was correlated with lower distance moved and shorter duration of movement. This was attributed to the fact that the increased velocity was caused by short movements and hence, only a short distance was moved. Therefore, they concluded that endpoints should be selected cautiously since swimming velocity might not completely represent other endpoints. Alternatively, Ingebretson and Masino [50] advocated the use of more than one endpoint as an integrated approach to maximize diagnostic capacity of behavioral activity. In the case of STC, two different endpoints were typically reported; frequency of STC and percentage of embryos showing spontaneous activity $[86,126]$. Since the latter endpoint only reveals the numbers of embryos showing STC, it may not be able to demonstrate effects that mainly affect the frequency of STC. For comparative assessment, the same endpoint parameter within a particular assay, i.e., distance moved for LMR and frequency for STC test should be used. 


\section{Rearing conditions}

Rearing conditions may also be considered as an extrinsic technical factor. However, since rearing conditions (raising and/or exposure in groups versus raising of individuals, choice of rearing temperature) are known to impact the development in general and behavior patterns, it was formally considered as intrinsic factor. Zellner et al. [127] observed that zebrafish embryos raised in groups were more active than those raised individually after the first 5 days of development. They proposed that rearing condition before behavior measurements could probably influence the effects of exposure to neuroactive substances. This seems consistent with the knowledge that swarm fish like zebrafish show shoaling and schooling and this (i.e., raising as a group) decreases their overall stress level under certain conditions [88]. This peer inclusion has also been reported to increase stimulation and may facilitate neuron connection [65]. A guidance document on fish rearing delineates the density as being important [64] but at the moment, it is not clear whether crowding is important for early stages of zebrafish and what group size would be the appropriate rearing size [83, 91]. However, when embryos were exposed to valproate by Zellner et al. [127], hyperactivity was recorded but no significant difference was observed for embryos raised singly or in-groups. Whether embryos were exposed singly or in-groups may also impact the results due to the requirement for different types of data analysis; e.g., individual tracking of movement is difficult when exposure is conducted with group of animals.

Rearing temperature represents an important factor as well since it influences the rate of development, and therefore the developmental stages at which behavior may be analyzed [57]. Other rearing conditions such as $\mathrm{pH}$ and salinity which could also impact the behavior analysis were not discussed due to insufficient data or lack of reporting. Therefore, it is recommended to report specific rearing conditions.

\section{Zebrafish strain}

In the literature, a discussion is ongoing whether different zebrafish strains may differ in their susceptibility to chemicals and how much is based on genetically based differences, physiological adaptations to cultivation or other parameters [28, 45]. For behavioral effects, De Esche et al. [27] investigated the influence of different strains ( $\mathrm{TL}$ and $\mathrm{AB}$ ) on locomotion in alternating light and dark periods. They found significant differences in distance moved but only in the dark periods. Lange et al. [63] also compared five different strains of $6 \mathrm{dpf}$ larvae (AB, TU, WIK, Casper and Ekkwill) and found that Casper and Ekkwill strains moved less than the other strains. They also compared $\mathrm{AB}$ strains from different laboratories and their results show that these $A B$ strains moved similar distances but had different swimming times. They concluded that the strain might influence locomotor activity of zebrafish. Similarly, Liu et al. [73] reported behavioral differences of strains (TL, TLAB and $\mathrm{AB}$ ) when measuring locomotion during abrupt changes in light cycles. Strain differences were also found in a study on survival and neurocranial effects of ethanol [75]. Further, strain differences have been reported during chemical exposure. 5D strain exposed to haloperidol showed hyperactivity, while the AB strain showed no effect [81]. These differences in strain behavior are probably related to genetic differences; albeit, the factors and differences leading to strain variability have not yet been identified. Hence, the possible influence of different strain or similar strain between different laboratories on zebrafish behavior should be considered.

\section{Technical (extrinsic) factors \\ Exposure concentration}

Exposure concentration is obviously one of the most important experimental parameters in toxicity studies. Hamm et al. [46] identified that the renewal or nonrenewal of exposure solution could influence toxicity testing, particularly in case of volatilization, degradation and/or adsorption to exposure vessels, resulting in a decline of exposure concentrations. The exposure concentration could represent a factor of high relevance in high-throughput studies that only examine a single, selected concentration for a given test chemical. Depending on the selected concentration, hypo- or hyperactivity may be provoked and this can have an impact on diagnostic patterns if obtained with a single or a limited range of concentrations. For example, ethanol causes hyperactivity at concentrations below $2 \%$ and hypoactivity at concentrations above $4 \%[27,52]$. Zebrafish exposed to the cholinesterase inhibitor paraoxon showed hyperactivity in a lower exposure range (31-500 nM) and became hypoactive with a 100-fold increase in concentrations $(3.1-50 \mu \mathrm{M})[86,126]$. The differential response may be associated with excitation of nerve signaling at lower concentrations due to acetylcholinesterase inhibition and seizure-driven paralysis at higher concentrations [111]. However, organophosphates have also been shown to affect axonal morphology of motor neurons at high concentrations (>500 nM) [123] and this could probably explain the hyperactivity observed at low concentrations rather than hypoactivity [126]. Therefore, behavioral tests should include a concentration-response analysis.

\section{Exposure duration}

In a STC study by Vliet et al. [118], exposure duration was reduced from $23 \mathrm{~h}(2-25 \mathrm{hpf})$ to $2 \mathrm{~h}(23-25 \mathrm{hpf})$ to 
eliminate false positives associated with malformations. This decreased the number of STC hits from 43 to 15. However, this decrease in hits could be compromised by a slow uptake of certain chemicals. Internal exposure analyses have indicated that for many chemicals, time to equilibrium with average internal concentrations can exceed $24 \mathrm{~h}$ depending on the compound's characteristics [13]. In behavior assays, that allow longer exposure durations such as the LMR, the same exposure concentration that causes hyperactivity in the STC may lead to axonal defects, malformations and/or paralysis resulting in hypoactivity in these long duration tests [61]. This could explain the opposite effect direction observed in STC tests (hyperactivity) and LMR tests (hypoactivity) for chlorpyrifos [120]. For similar reasons, differences in the effect direction (hypo- or hyperactivity) may also be observed within the same assay when different exposure durations are used. For instance, Leuthold et al. [68] have applied a 24-h exposure regime in the LMR-L/D and differences to studies conducted with longer exposure durations may be associated with the factor described above. This means that significant differences in exposure duration could influence the internal concentration of a chemical. Hence-if that is a possible confounding factor for the goal of the experiment, behavioral test designs should ideally measure or try to model the toxicokinetics/toxicodynamics of the chemical for appropriate result interpretation, e.g., short duration for identifying neuroactivity MoA, while long duration may be preferred for assessing developmental neurotoxicity.

\section{Duration of behavior analysis}

Zebrafish embryos are sensitive to alternating light/dark periods. Non-exposed zebrafish were found to be more active in dark than light periods. Therefore, by purpose, various studies have utilized cycles of light and dark periods to improve detection of hypo- and hyperactivity (e.g., [52, 61, 68, 129]). However, the dynamics of analysis duration has been shown to affect test outcome. Exposing $6 \mathrm{dpf}$ larvae after a dark acclimation period of $10 \mathrm{~min}$ and a subsequent extended $(40 \mathrm{~min}$ ) period in light (or dark) showed contrasting behavior-activity increased to a maximum in the 10 -min dark acclimation period, followed by a decline which continued to either a stable low level in the subsequent dark period or to a stable high level in the light period [76]. Interestingly, in a light/ dark preference test by Steenbergen et al. [110], zebrafish prefer light and the increased movement in the dark is hypothesized to relate to a behavior such as escaping from a predator [27]. Alternately, it was discussed that foraging in zebrafish depends on their visual system to find food, and therefore increased activity in the dark may be related to a light-searching behavior [48]. For toxicity tests, the basal activities during light and dark periods are important to detect hypo- and hyperactivity. While it might be difficult to detect hypoactivity in light periods given the low activity level, detection of hyperactivity could be more relevant during this period. The duration and frequency of light/dark cycles could also be of high relevance in a toxicity testing setup. Dark acclimation of zebrafish (10 min), followed by different light periods of either 5 or $15 \mathrm{~min}$, showed a higher magnitude of increased activity in the subsequent dark period for the larvae exposed to 15-min light than that of $5 \mathrm{~min}$ [76]. Different dark acclimation periods of 10 and $20 \mathrm{~min}$ did not affect activity in subsequent light and dark periods. Another study by Liu et al. [73] found that analysis of short periods of $30 \mathrm{~s}$ before and after light/dark transition amplified behavior changes. Taken together, these results suggest that extended periods of light or dark can impact the activity of zebrafish embryos and hence may affect the sensitivity and outcome of the study.

\section{Exposure well size}

Velki et al. [117] compared the total distance moved for zebrafish embryo/larvae exposed in 24- or 96-well plates in the dark period. They reported an average distance of 600-700 mm moved in 24-well plates, whereas those in 96-well plates moved $40 \%$ less. Similarly, Padilla et al. [82] found that larval movement was approximately 4 times higher in 24-well than in 96-well plates and distance moved in 48-well plate was not statistically different from that of 96-well plate. They elucidated that the distance moved is mainly influenced by the circumference of the well rather than the area since embryos tend to swim more around the circumference of the well [20]. In contrast, Ingebretson and Masino [50] found no difference in distance moved when different well diameters of 12, 20 and $30 \mathrm{~mm}$ were compared [approximately referring to the diameter of $48-(10.9 \mathrm{~mm}), 24-(15.5 \mathrm{~mm})$ and 6-well plates $(35 \mathrm{~mm})$. A potential bias by rearing conditions can probably be excluded since embryos were raised and tested in the same wells in Padilla et al. [82] but transferred to well plates after exposure for behavioral analysis in all other studies.

From the analyzed literature, it is not yet clear if the decreased distance in smaller wells is associated with a lower sensitivity of the assay. For test reproducibility and uniformity, it is recommended to use exposure wells of same dimension. As an alternative, one could conduct experiments in different well sizes to ensure maximal confidence of the sensitivity of the assay used.

\section{Light conditions}

Padilla et al. [82] investigated the influence of light intensity on the behavior of $6 \mathrm{dpf}$ zebrafish larvae. They found 
an increased activity when light levels were decreased and this increasedactivity was dependent on the magnitude of the decreased light intensity. Therefore, light intensity, duration, and sequence of photo-stimuli should be recorded in different experimental setups and their impact on the effects obtained by exposure of chemicals should be investigated.

\section{Material used for exposure vessels}

The sorption of lipophilic substances to plastic material used for exposure is well known $[36,104]$. Sorption could decrease the exposure concentration leading to underestimation of effects if based on nominal concentrations. Raftery et al. [86] exposed embryos to the highly hydrophobic glutamate channel blocker-abamectin. They found an effect concentration for the spontaneous tail coiling (STC, conducted in 384-well plates) which is 12 -fold higher than that reported in a subsequent study [87] in which embryos were exposed in glass beakers (both used nominal concentrations). Similarly, Vliet et al. [118] who also exposed embryos to abamectin in 384-well plates found an effect concentration which is 6 fold higher than that observed in Raftery and Volz [87]. Beside the test container material, the ratio of surface area to volume could also induce variability. The reported effect concentration for emamectin is over 40 times lower when embryos were exposed in 24-well plates [121] compared to exposure in 384-well plates [86]. This could be the result of higher well absorption area with respect to volume in 384-well plates leading to higher adsorption. Hence, as would be appropriate for any other test and endpoint, the sorption of chemicals to exposure vessels should be considered by: (1) determining or predicting the real exposure concentration; and (2) estimating a possible loss of the chemical instead of using nominal concentrations only [35, 43, 44].

\section{Solvent concentration}

Solvents such as dimethyl sulfoxide (DMSO) are often used to accelerate dissolutions or to store stock solution for toxicity tests. At high concentrations, solvents may also impact the uptake of chemicals and provoke toxicity. Therefore, OECD guidance for aquatic toxicity tests recommends using a maximum solvent concentration of $0.01 \%(\mathrm{v} / \mathrm{v})$. In concordance with these recommendations, Kais et al. [55] reported that DMSO concentration of $0.1 \%$ and $1 \%$ increased uptake of hydrophobic dyes into zebrafish embryos, while $0.01 \%$ had no effect. DMSO has also been found to increase the movement of 24 and 144 hpf embryo/larvae at 0.1 and $0.01 \%$, respectively $[19,49]$. These results showed that DMSO may be a source of confounding effects in behavioral tests. It is highly recommended to avoid solvents, but if necessary, a solvent control should always be utilized and a range of solvent concentrations could be tested to characterize and exclude possible confounding effects.

\section{Summary of influence of experimental parameters}

The studies cited above showed the possible impact of different biological and technical experimental parameters on the behavioral response of zebrafish exposed to a chemical. Thus, experimental parameters may influence changes in behavior induced by chemicals. We ranked the importance of experimental parameters based on the frequency at which they explained inconsistencies (risk of bias factors) in chemicals as follows (Fig. 2): exposure duration (11) > exposure concentration (10) > endpoint parameter $(8)>$ developmental stage $(7)>$ light conditions $(2)>$ material used for exposure vessel $(1)=$ exposure well size $(1)=$ duration of behavior analysis $(1)=$ zebrafish strain (1). This ranking does not consider the behavioral test method as a factor.

Depending on the goal of the research question, the reproducibility of behavioral tests may be improved by developing protocols with harmonized experimental parameters. However, while there is evidence for the impact of intrinsic and extrinsic parameters affecting behavior, only few studies have conducted a systematic assessment on how this may impact the detection of chemical effects. One of such studies found that developmental stage, light conditions during rearing and size of well plate affected the locomotor response of zebrafish larvae exposed to bisphenol A and tetrabromobisphenol A [38]. Although the evidence that effects of exposure to chemicals may not be detected due to the choice of experimental parameters is not clear, the observed effects in unexposed groups are sufficient to motivate the harmonization of behavioral tests for specific hypotheses.

\section{Comparative assessment of observed activity (hypo- and hyperactivity) in zebrafish and expected activity based on mode of action of chemicals}

The review above describes the influence of biological and technical factors on behavioral assays. This outcome was then utilized to prioritize which experimental factors may be considered to influence results when comparing observed activity in zebrafish to expected activity based on relation to the mode of action (MoA). Therefore, a collection of publications was analyzed with respect to chemical type, mode of action, effect concentration (for hyper- and hypoactivity), endpoint parameter, exposure duration, duration of behavior analysis, exposure well size, developmental stage at exposure and zebrafish strain used in zebrafish embryo behavior tests. Figure 1 shows the flowchart that 


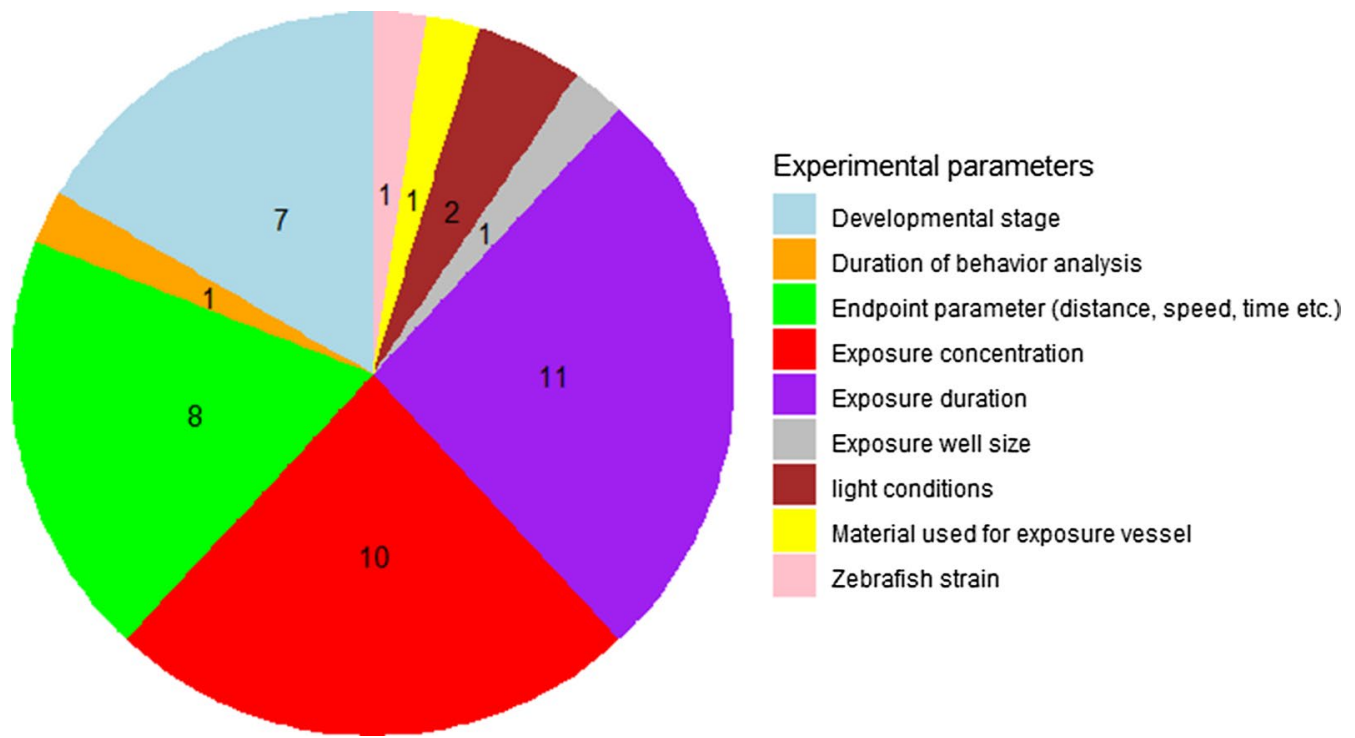

Fig. 2 Pie chart showing the weight of influence of the analyzed experimental parameters. The numbers within each pie represents the weight of each parameter. The weight analysis was done by ranking each parameter according to the number of times it occurred as an assumed reason (risk of bias) to explain inconsistencies within chemical comparison. The risk of bias factors are recorded in Table 3 for each chemical

summarizes how the collected papers were processed. In the first step, 885 studies were obtained in the "web of science" search and these were reduced to 111 studies (Additional file 2: Excel $^{\circledR}$ sheet 1) after a manual abstract screening process based on the exclusion criteria. Second, the study hits were transformed through a series of aggregation and regrouping processes (to exclude chemicals with less than 3 study hits) in KNIME $^{\circledR}$ to obtain 31 chemical hits (Additional file 2: Excel $^{\circledR}$ sheet 2). The chemical hits were subjected to a further exclusion criterion to eliminate non-pharmaceutical and non-pesticide chemicals and to retain only chemicals with well described mode of action. Finally, a total of 18 chemical hits were analyzed in this review (Additional file 2: $\operatorname{Excel}^{\circledR}$ sheet 3).

To analyze the association of the expected mode of action of a chemical to its observed effects (i.e., hypo- or hyperactivity) in zebrafish embryos, a comparison was done in relation to individual substances with known neuroactive mode of action or substance classes with different neuroactive mode of action. Furthermore different behavioral test methods were compared for the same compounds.

\section{Comparison of individual substances with known neuroactive mode of action}

Neuroactive substances with at least three entries in the established literature collection were compared to estimate whether in zebrafish embryos:
1. Similar behavioral methods resulted in a consistent behavioral response across studies with regard to anticipated activity (hypo- or hyperactivity),

2. Different methods (STC, PMR, LMR, LMR-L/D) gave consistent anticipated activity (hypo- or hyperactivity),

3. The observed activity was consistent with the anticipated activity regardless of the method used,

4. The respective effect concentrations in the different studies are similar-in cases when hypo- or hyperactivity is consistent between the studies.

Eighteen different comparative assessments were conducted. These were organized into three groups according to the expected effect, based on the MoAhyperactive, hypoactive and unclear. We discussed all 18 substances but only show herepentylenetetrazole (PTZ) and abamectin as representative substances for hyperactivity and hypoactivity, respectively. Detailed discussions and corresponding figures for the remaining substances can be found in Additional file 1. Nonetheless, results for all substances are summarized in Table 3. A comprehensive overview of all data is shown in Fig. 3. The effect concentrations for all 18 chemicals span over 8 orders of magnitude and individual chemicals range over 2-3 orders of magnitude. Such high variation in effect concentrations reveals the heterogeneity of the results obtained which may be attributed to the use of different experimental protocols and parameters. For instance, an inconsistent activity trend (hyper- or hypoactivity) can be 


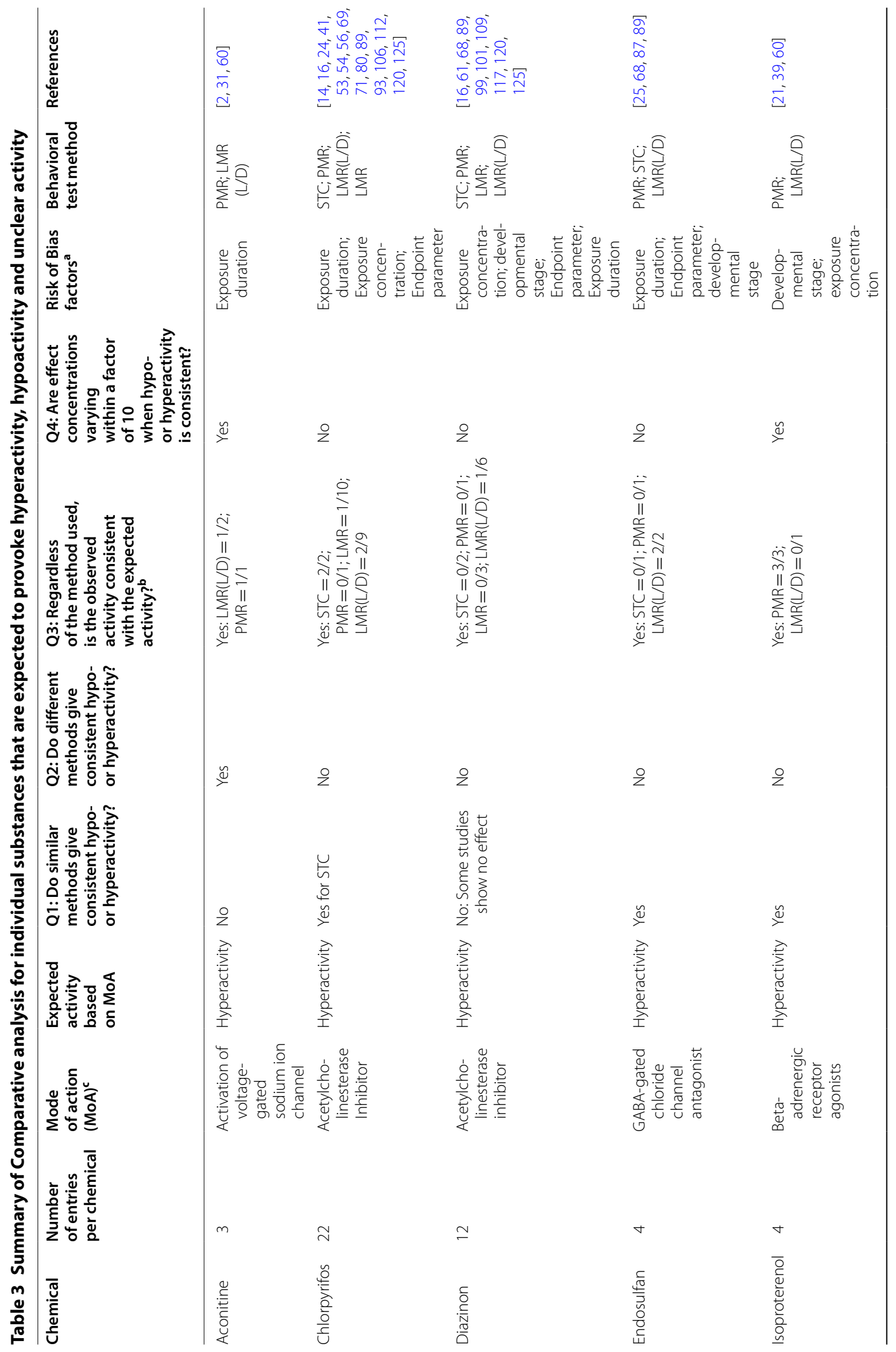




\begin{tabular}{|c|c|c|c|c|c|}
\hline 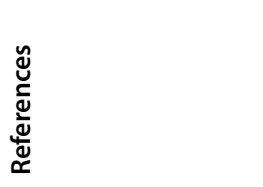 & 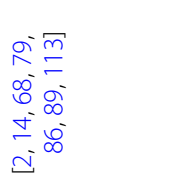 & 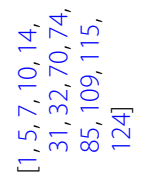 & 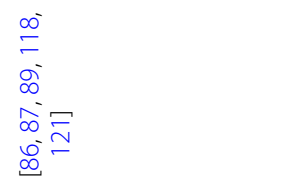 & 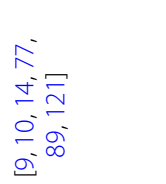 & 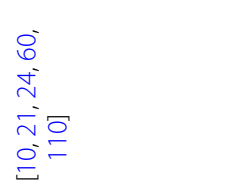 \\
\hline 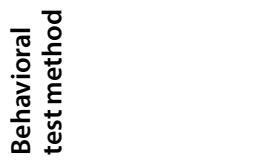 & 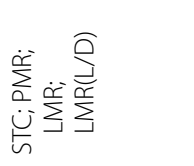 & 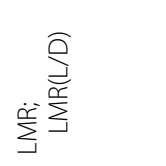 & 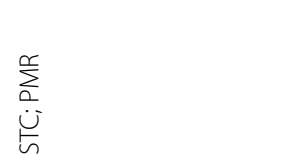 & 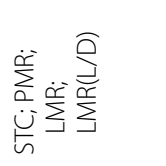 & 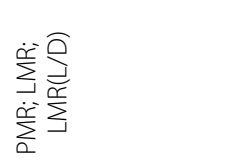 \\
\hline 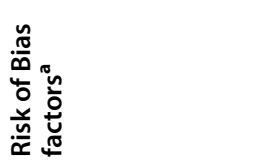 & 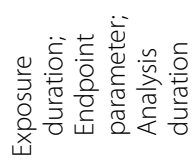 & 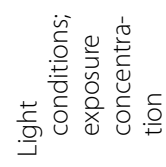 & 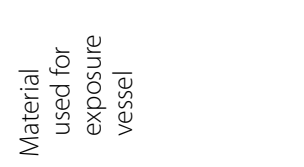 & 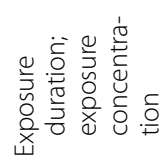 & 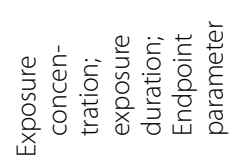 \\
\hline 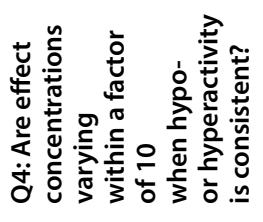 & $\stackrel{o}{z}$ & $\stackrel{\tilde{\nu}}{\sim}$ & $\frac{o}{z}$ & $\stackrel{\tilde{u}}{\searrow}$ & $\stackrel{o}{z}$ \\
\hline 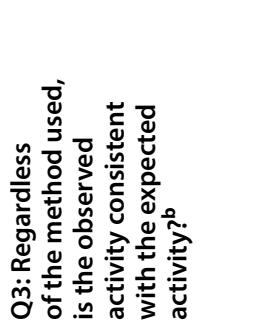 & 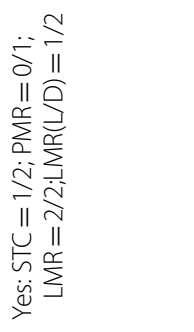 & 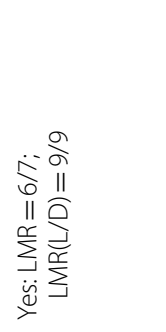 & 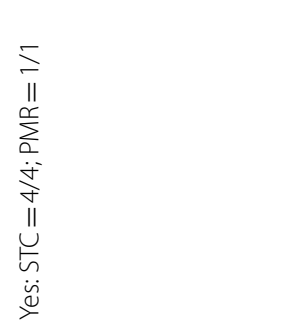 & 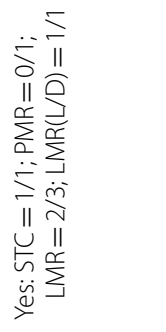 & 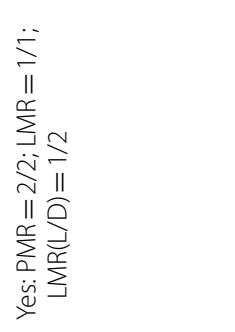 \\
\hline 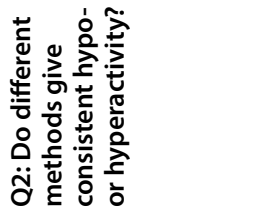 & $\stackrel{\breve{g}}{=}$ & $\stackrel{\tilde{\Perp}}{x}$ & $\stackrel{\check{\Perp}}{\varnothing}$ & $\stackrel{\check{\Perp}}{\nearrow}$ & $\stackrel{\varpi}{\nsim}$ \\
\hline 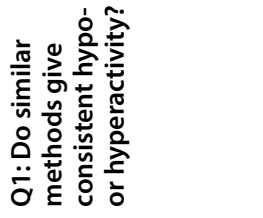 & 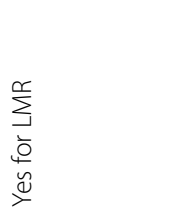 & $\stackrel{\dddot{y}}{x}$ & $\stackrel{\varpi}{\nsim}$ & 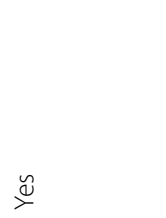 & $\stackrel{\oiiint}{\simeq}$ \\
\hline 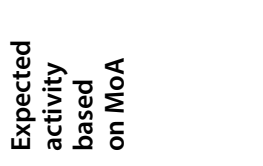 & 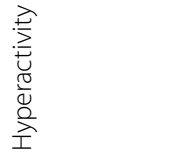 & 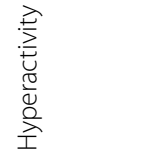 & 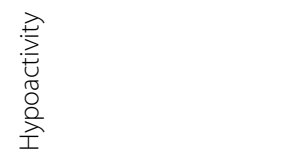 & 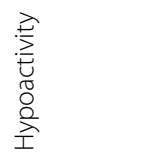 & 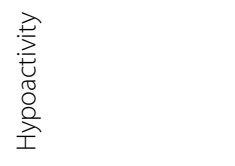 \\
\hline 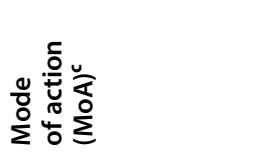 & 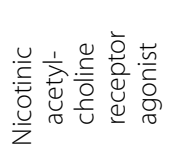 & 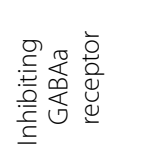 & 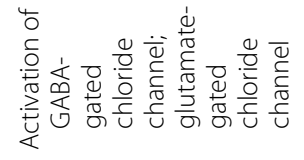 & 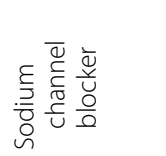 & 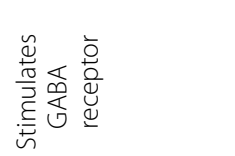 \\
\hline 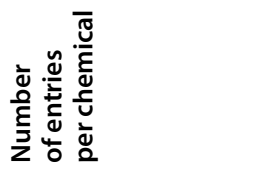 & $\wedge$ & $\stackrel{\circ}{\circ}$ & in & 0 & in \\
\hline & 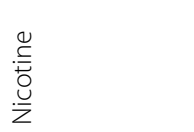 & 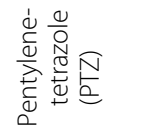 & 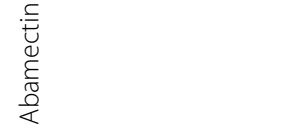 & 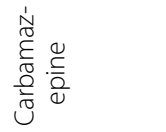 & 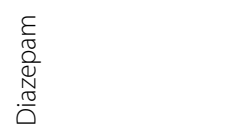 \\
\hline
\end{tabular}




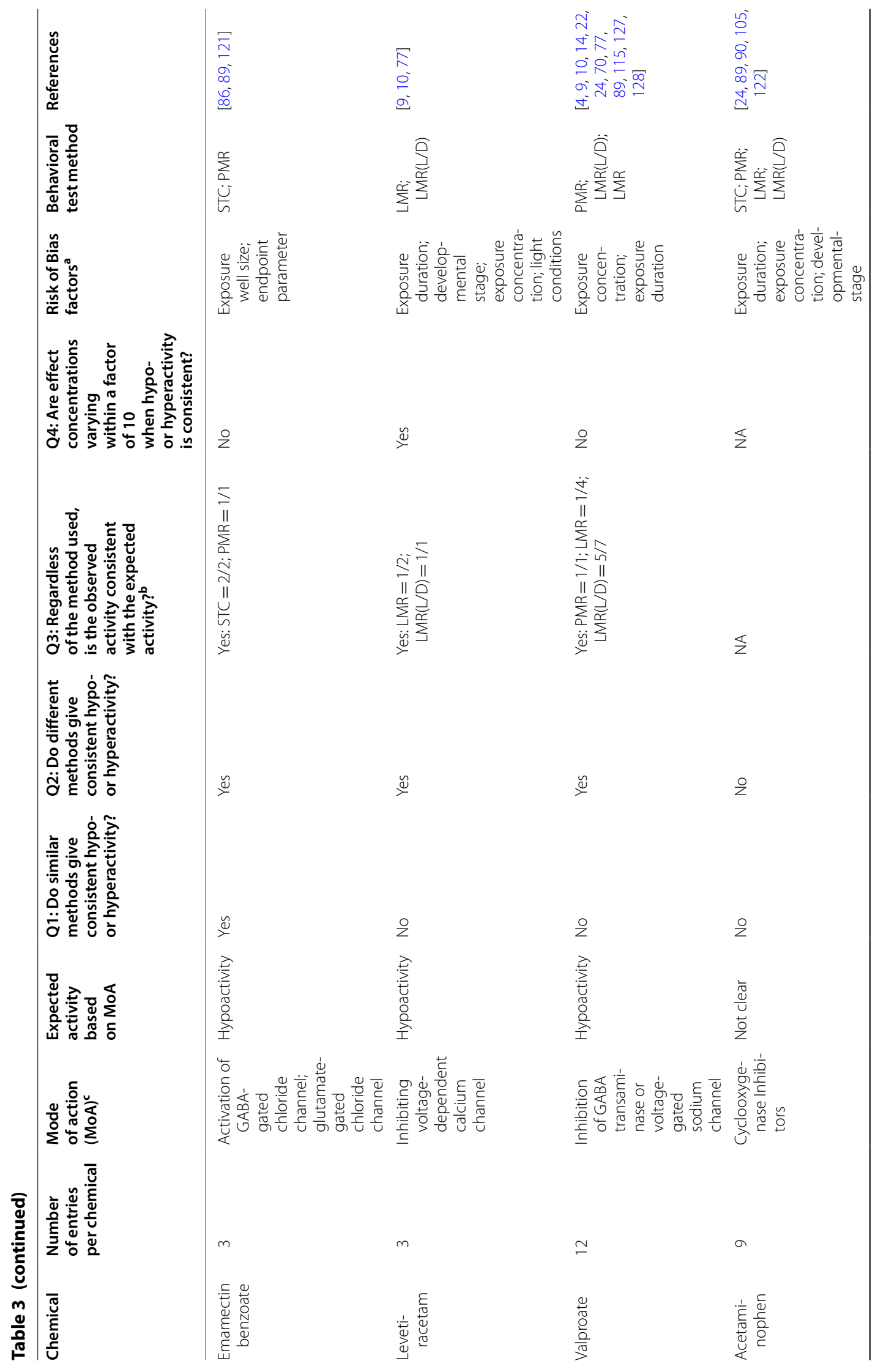




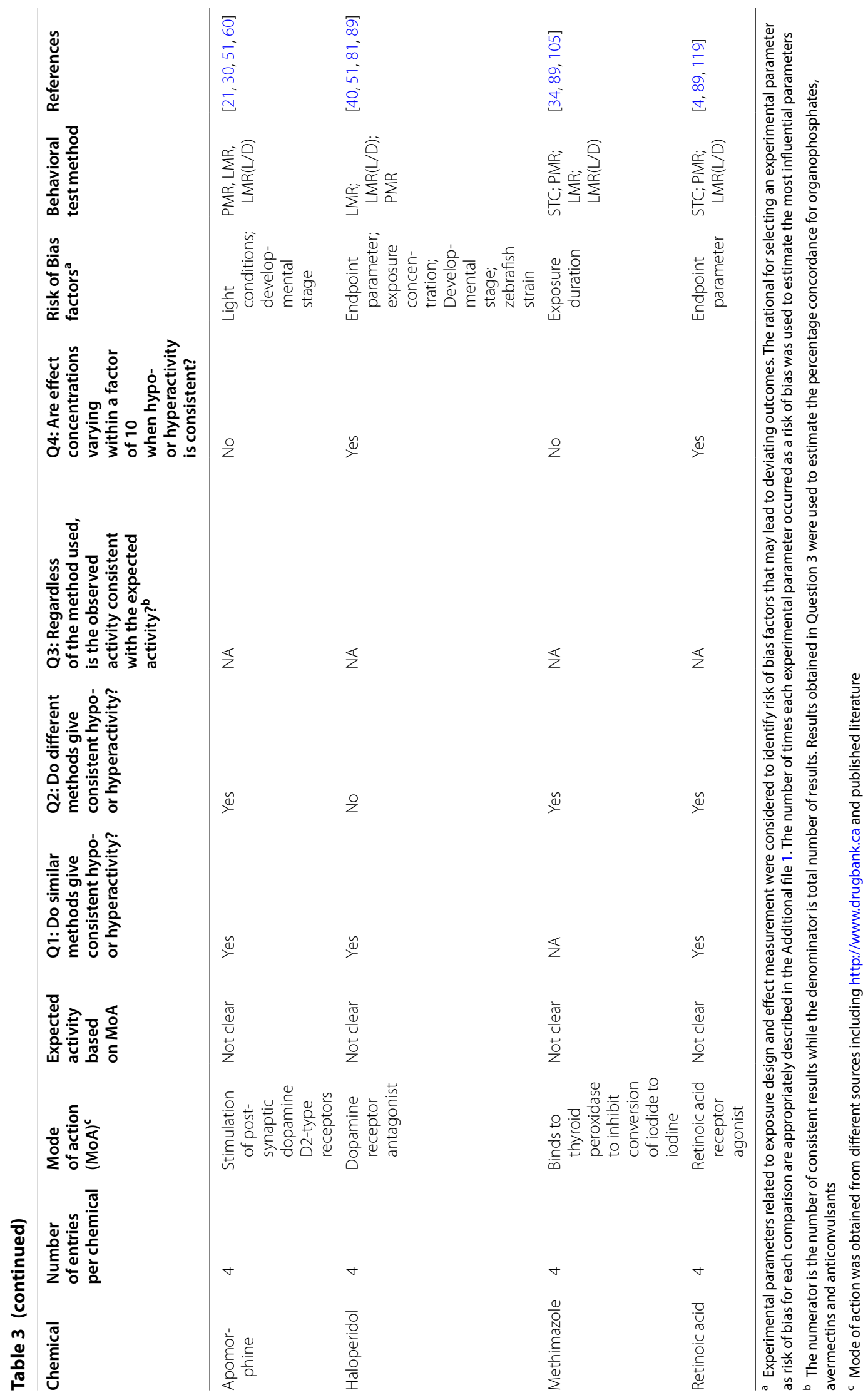




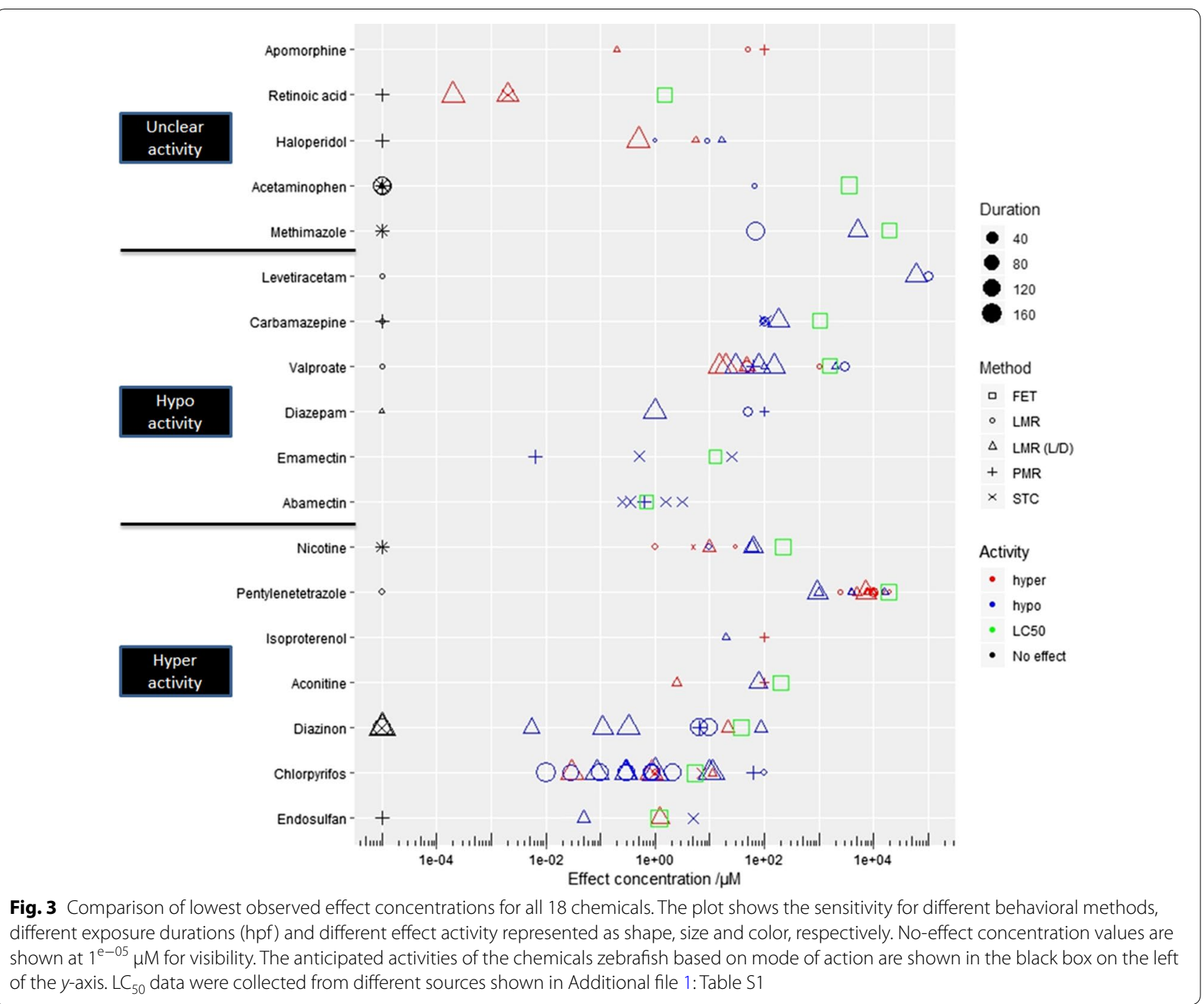

seen for chemicals expected to cause hyperactivity and this is probably influenced by high variability of exposure duration (Fig. 3).

\section{Abamectin}

Abamectin is an avermectin insecticide expected to cause hypoactivity by activating GABA-gated chloride channel [17]. Five studies were compared. All studies reported hypoactivity (Fig. 4). Effect concentration for hypoactivity reported in all studies is within a factor of $10(0.36-3.13 \mu \mathrm{M})$ except the STC study by Raftery and Volz [87] which reported an effect at $0.25 \mu \mathrm{M}$. This lower effect concentration could be due to conducting exposure in glass beakers instead of plastic well plates as exposure vessel. Abamectin is highly hydrophobic $\left(\log D_{\mathrm{pH} 7.4(\mathrm{ACD} /}\right.$ Labs) of 5.85) and hence has higher affinity to bind to plastic than glass. Therefore, abamectin may be highly bioavailable to the embryos in a glass container leading to effects occurring at lower concentration.

\section{Pentylenetetrazole (PTZ)}

Pentylenetetrazole is a convulsant drug and it is expected to cause hyperactivity by binding to GABA receptors [108]. Sixteen studies were compared. PTZ showed hyperactivity effects in all the studies except the LMR study by Bugel and Tanguay [14] which reported no effect (Fig. 4). However, in this study, exposure concentrations 2.5-fold below the effect concentration of the other studies were used and this was probably below the effective range of PTZ. Even though the effect concentrations for hyperactivity were within a factor of 10 in all studies, hypoactivity was also reported, at different concentrations and light periods, as an additional effect to hyperactivity in some LMR-L/D studies. The effect of PTZ may be enhanced under alternating light-dark 


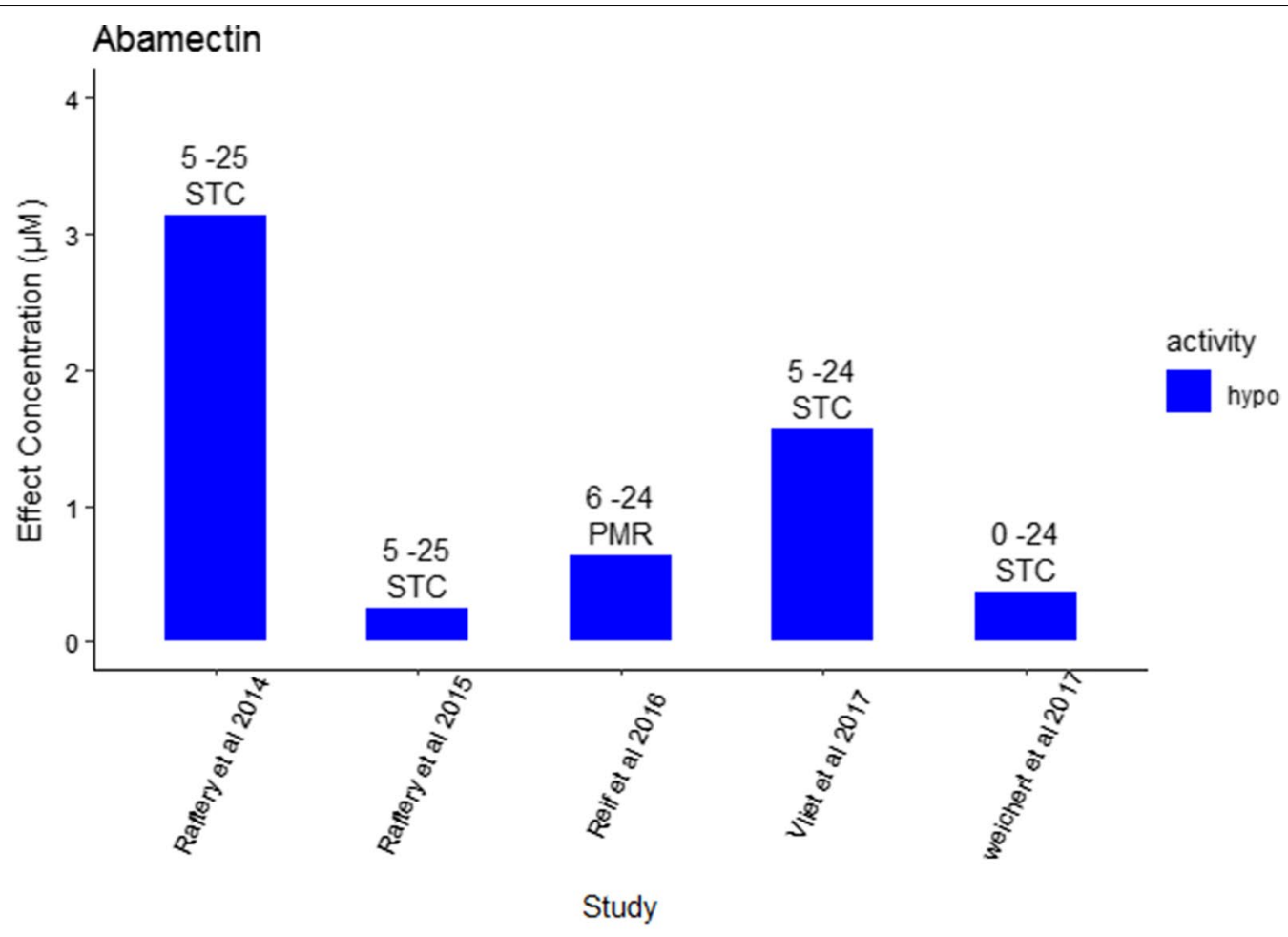

Pentelenetetrazole (PTZ)

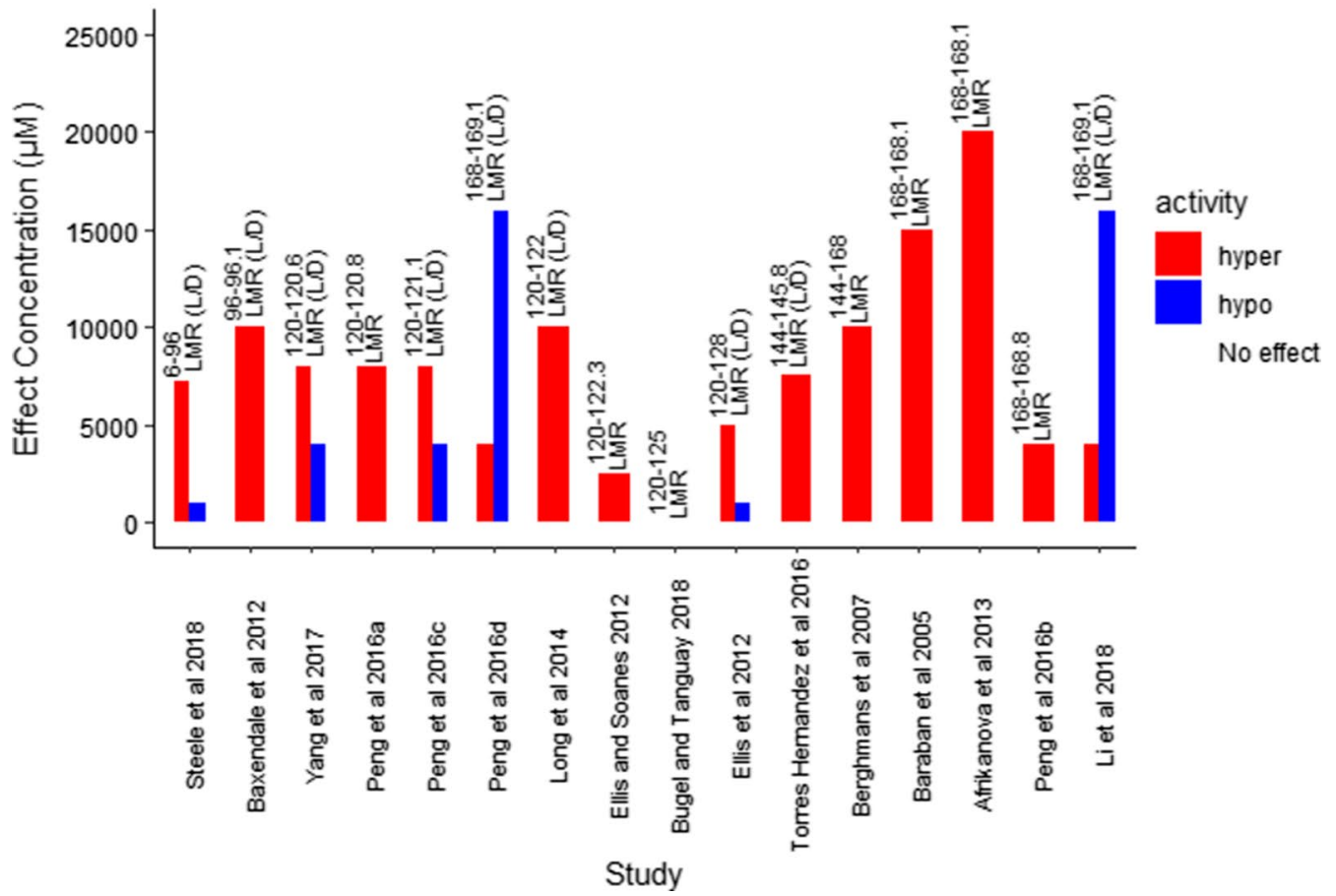

Fig. 4 Effect concentrations for pentylenetetrazole and abamectin that are expected to provoke hyperactivity and hypoactivity, respectively. Bars show the magnitude of the effect concentrations which represents lowest effect concentrations as deduced from each study. When there is no bar, it indicates no effect observed within the tested concentration range. When two different bars are depicted for one study, it indicates effect concentrations for both hypo- and hyperactivity. The text written on top of each bar represents the behavioral test method while the numbers represents the exposure duration (hpf). References for each study can also be found in Additional file 2: Excel sheet 3 
periods and PTZ has been reported to cause a reversal of the observed activity in control treatment, i.e., higher activity in dark and lower activity in light phase for nonexposed embryos [31, 115]. Consequently, it is likely that PTZ induces a differentialresponse in light and dark phases and this effect is only observed under alternating light conditions. Hence, the use of different light conditions during measurement could be a limiting factor for comparing the output from different behavior methods. Nevertheless, alternating light conditions could give important insights on how a substance modulates behavior.

\section{Comparison of substance classes with same/similar mode of action}

Neuroactive chemicals which emerge from the same chemical class can be anticipated to exhibit similar mode of action. The aim of this comparison is to evaluate the consistency of the observed activity of chemicals in zebrafish to the expected activity within a certain class of chemicals (organophosphates, avermectins and anticonvulsant drugs) e.g., do all chemicals within a class conform to the expected mode of action (hypo- or hyperactivity) in zebrafish embryos?

\section{Organophosphates}

Organophosphates act by inhibiting the acetylcholinesterase enzyme (AChE) which breaks down acetylcholine, and therefore keeps the acetylcholine-gated sodium channels open for more ions to flow into the cell leading to an action potential [17]. Hence, it is expected for organophosphates to cause hyperactivity in zebrafish at lower concentrations but hypoactivity at higher concentrations due to over-excitation resulting in paralysis induced by abnormal mechanical stress [111] or axonal deformation [123]. Additionally, the interaction of exposure concentration and duration plays a major role. For instance, chlorpyrifos caused hyperactivity at a low concentration of $0.03 \mu \mathrm{M}$ and long duration of $120 \mathrm{~h}$ in an LMR-L/D study (Fig. 5). Inversely, a high concentration of $11 \mu \mathrm{M}$ and short duration of $4 \mathrm{~h}$ also resulted in hyperactivity [53, 80]. A summary of the data for organophosphates (Chlorpyrifos and Diazinon) is shown in Fig. 5 and in Additional file 1. Although hyperactivity is expected for lower test concentrations, it was only observed in $18 \%$ of the studies. The percentage concordance of observed to expected activity was estimated to be $50(n=4), 0(n=2), 7.7(n=13)$ and $20(n=15) \%$ for STC, PMR, LMR and LMR-L/D, respectively (number of studies per method in parenthesis). Considering that STC has the highest percentage of consistency, we could deduce that STC may be the most-sensitive method to detect the hyperactive effect of organophosphates. This could be due to the assumed capability of the STC to measure basic response of the primary motor neurons $[92,98]$ rather than the secondary neurons measured in LMR. Many organophosphates require biotransformation for highest inhibition capacity [18], and a limited biotransformation in embryos would represent a confounding factor and might interfere with the detection of

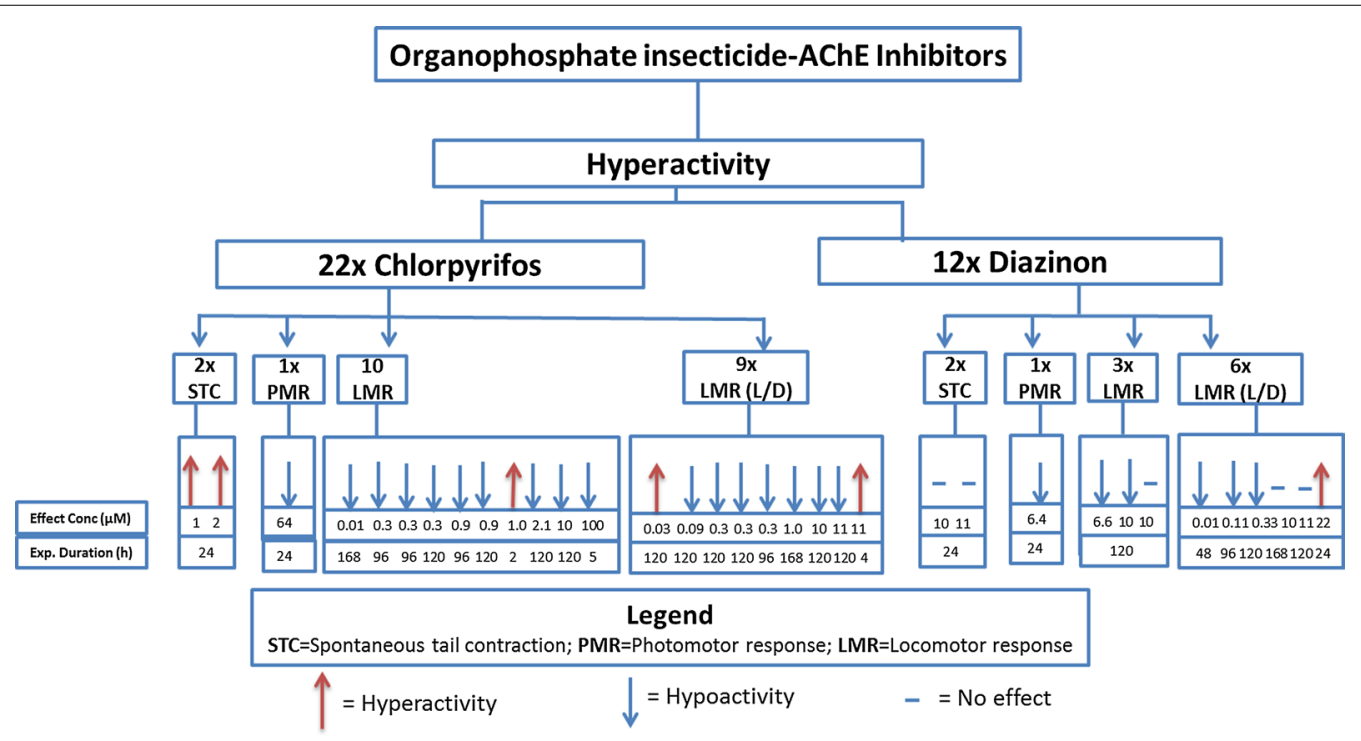

Fig. 5 Flowchart showing the consistency of two organophosphates to hyperactivity. The number in front of the substance and the method shows the total number of chemical hits and the chemical hits per method, respectively. The arrows indicate hyper-, hypo- or no activity. The numbers below each arrow indicates the effect concentration in $\mu \mathrm{mol} / \mathrm{L}$. The arrows are organised with increasing effect concentrations from left to right 
behavioral effects in early embryo stages. Interestingly, the LMR-L/D study by Leuthold et al. [68] was the only one that showed hyperactivity for diazinon (Fig. 5). This could be due to the use of a combination of older developmental stage of $96 \mathrm{hpf}$ (with potential higher biotransformation) and a short exposure duration of $24 \mathrm{~h}$ (with potential no over-excitation or paralysis effect). However, not only the oxon-metabolite but also the parent compound of chlorpyrifos induced hyperactivity in the STC at an earlier developmental stage of $24 \mathrm{hpf}$ (with potential limited biotransformation). This suggests the influential AChE inhibiting activity of chlorpyrifos or a high efficacy of the low amount of the oxon-metabolite resulting from the limited biotransformation [120, 121]. Reif et al. [89] also found a relatively lower effect concentration $(0.64 \mu \mathrm{M})$ for chlorpyrifos-oxon compared to chlorpyrifos $(64 \mu \mathrm{M})$ and this supports the limited biotransformation of chlorpyrifos in younger developmental stages. Hence, for neuroactive compounds such as organophosphates which require bioactivation, the stage-dependent bioactivation or the internal concentrations related to the exposure time may influence the effect concentrations.

\section{Avermectins}

Avermectins act by activating the GABA-gated chloride channel and/or glutamate-gated chloride channel leading to an inhibitory potential and hence hypoactivity [17]. Both avermectin chemicals considered in this study, emamectin and abamectin, showed hypoactivity which is consistent with their mode of action. A summary of the data for avermectins shows that $100 \%$ hypoactivity effect was reported. Percentage concordance of observed to expected activity was estimated to be $100(n=6)$ and $100(n=2) \%$ for STC and PMR, respectively (number of studies in parenthesis). This shows that avermectins can be reasonably detected in short-duration embryo tests. See Additional file 1 for more details on abamectin and emamectin.

\section{Anticonvulsant drugs}

Anticonvulsants are a class of drugs used for controlling seizure activity. They propagate their action on the nervous system via different mechanisms including: (1) Blockage of the sodium-gated channels. (2) Indirect or direct enhancement of inhibitory GABA neurotransmission. (3) Inhibition of excitatory glutamatergic neurotransmission [107]. Based on their mode of action, it is expected that anticonvulsants will cause hypoactivity in zebrafish embryo. A summary of the data for anticonvulsants (Fig. 6) shows that despite using different methods, 62\% hypoactivity effect was reported. Percentage concordance of observed to expected activity was estimated to be $100(n=1), 75(n=4), 50(n=10)$ and $64(n=11) \%$ for STC, PMR, LMR and LMR-L/D, respectively (number of studies in parenthesis). STC was not considered since only one study was found. Hence, we can deduce that PMR and LMR-L/D may be the most-sensitive method to detect anticonvulsants and this could be due to the light stimulation utilized in both methods which may interact with complex nervous processes in the brain.

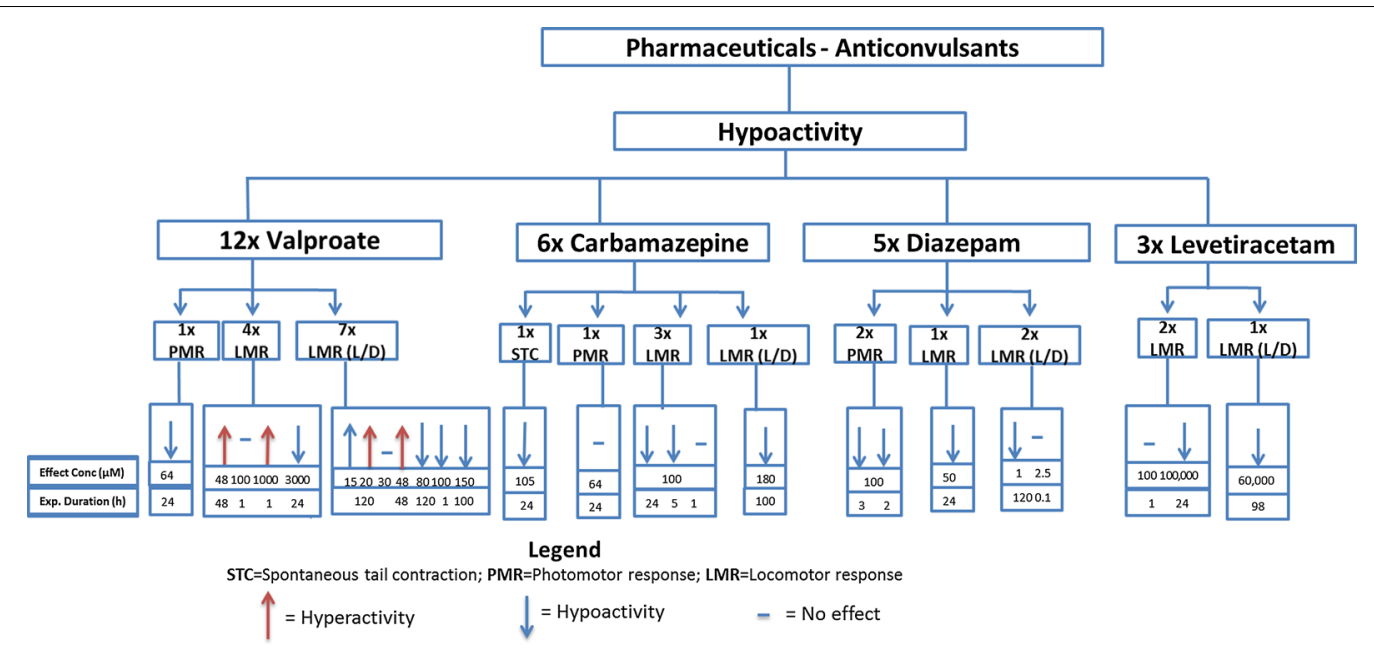

Fig. 6 Flowchart showing the consistency of anticonvulsants to hypoactivity. The number before the chemical and the method shows the total number of chemical hits and the chemical hits per method. The arrows indicate hyper-, hypo or no activity. The numbers after each arrow indicates the effect concentration in $\mu \mathrm{mol} / \mathrm{L}$. The arrows are organized with increasing effect concentrations from left to right 


\section{Comparison of different behavioral methods}

In the last part of this results section, we tried to estimate whether different behavioral measurement methods or contrasting experimental parameters give similar results. For that we identified case studies that reported different methods or setups for the same chemical or different chemicals. Here, the goal is to compare two behavioral methods using substance(s) tested in both methods.

\section{Effects of abamectin in the STC}

\section{Case study: Raftery et al. [86, 87], Vliet et al. [118], Weichert}

et al. [121]

For abamectin, an enhancer of GABA- or glutamategated chloride channel, five different studies have reported the effects in the STC (Fig. 4). While the material used for exposure vessels varied to some extent, developmental stage, exposure duration and duration of behavior analysis were very similar for these studies. The effect concentrations of the different studies were within a factor of 10 (except[87]) and in all studies hypoactivity was observed. As described earlier (see comparison of individual substances), the minor differences in effect concentrations may be attributed to the use of different exposure vessels. For instance, the lowest effect concentration $(0.25 \mu \mathrm{M})$ was reported in a study that used glass exposure vessels [87]. Moreover, exposures in 24-well plates, with lower surface area, resulted in lower effect concentrations compared to 384-well plates [86, 118]. Abamectin has a high log Kow of 5.85 and this suggests that the lower effect concentrations in glass and 24-well vessels could be due to higher bioavailability. This may be attributed to higher adsorption to plastic wells (relative to glass) and especially 384-well plates (relative to 24-well plates). In general, the results of STC were in line with the mode of action and largely consistent between studies and differences attributed to the adsorption of the compound to exposure vessels. However, Vliet et al. [118] concluded that STC might not be capable of distinguishing between modes of action because they observed only hypoactivity even for chemicals with hyperactive MoA. Possible reasons for their observed hypoactivity include the use of a single exposure concentration and use of a different endpoint (percentage of embryos showing STC), which might be inherently biased (see "Endpoint parameter" section for more details).

\section{Comparison of STC and PMR}

Case study: Fipronil, emamectin benzoate, carbamazepine,

\section{abamectin}

Comparison of results between STC and PMR for some chemicals showed a lack of consistency in the observed effect concentrations. There are two exceptions. First, abamectin comparison in which the STC studies $[87,121]$ showed similar effect concentrations to another PMR study [89]. Second, behavioral effects were observed for carbamazepine in the STC test [121] at a concentration not tested in the PMR [89]. The chemicals that show inconsistencies include; fipronil, chlorpyrifos and emamectin. For fipronil, an effect concentration of $25 \mu \mathrm{M}$ was reported for the STC study by Raftery and Volz [87], while the PMR study by Reif et al. [89] showed no effect at concentrations up to $64 \mu \mathrm{M}$. This difference could be due to the use of plastic well plates in the study of Reif et al. [89] leading to a possible adsorption and hence decreased exposure and effect concentrations. For emamectin, the STC study by Weichert et al. [121] reported a hypoactivity effect at $1.03 \mu \mathrm{M}$, while the PMR study by Reif et al. [89] reported 0.0064 $\mu \mathrm{M}$. Even though STC and PMR have many similar experimental parameters, there is a major difference in their endpoint parameter. While PMR measures movement of the whole embryo under light stimulation, STC only measures the spontaneous tail contractions. This difference could lead to major differences in the behavioral outcome of both tests (see Additional file 1 for more details).

\section{STC/LMR comparison}

Case study: Chlorpyrifos in Selderslaghs et al. [105, 106]

Selderslaghs et al. $[105,106]$ exposed zebrafish embryos to different chemicals and both the LMR and STC were conducted to identify neuroactive effects or effects on neurodevelopment. STC captured effects of more substances and this sensitivity difference could be due to a different range of exposure concentrations. Higher exposure concentrations were used for the STC test because exposure concentrations were based on the highest non teratogenic concentration estimated at exposure durations of 24 and $144 \mathrm{~h}$, respectively [105]. Even though, different concentration ranges were used, effects were observed at an overlapping concentration of $1.8 \mu \mathrm{M}$ and $2.1 \mu \mathrm{M}$ for chlorpyrifos in STC and LMR, respectively [106]. However, the effects were not consistent-hyperactivity in STC and hypoactivity in LMR. This activity difference may be due to the influence of exposure duration (steady state not reached in shorter exposure), and/ or developmental stage-dependent metabolic activation. As discussed earlier (see sections on exposure duration and organophosphates), longer exposure might increase the internal concentration of the substance and thus increase effects. Vice versa, a short exposure might need higher external and thus internal concentrations to get the same effects. In the case of chlorpyrifos, one could assume that the longer exposure caused over-excitation of acetylcholine receptors due to the irreversible inhibition of $\mathrm{AChE}$ and thus the resulting paralysis translates 
to hypoactivity. Therefore, similar exposure durations should be used for a comparison of the sensitivity of both assays (albeit differences in toxicokinetics and targeted receptors of the tested developmental stages may still apply). Moreover, STC measures the response of the primary motor neurons in the embryo while LMR measures the response of both primary and secondary motor neurons in free swimming embryo $[42,126]$. Hence, differences in the results could occur because these assays partially target different neuronal structures.

\section{PMR/PMR comparison}

\section{Case study: Isoproterenol, apomorphine and diazepam} in Kokel et al. [60] and Copmans et al. [21]

Kokel et al. [60] and Copmans et al. [21] were selected for this comparison because crucial experimental parameters including exposure age, exposure duration and analysis time were similar. Also, three chemicals namely isoproterenol, apomorphine and diazepam were tested in both studies (see Additional file 1). Despite the fact that a single concentration was tested in both studies, similar effect concentrations and activity were obtained. Embryos were hyperactive to isoproterenol and apomorphine, while hypoactivity was observed for diazepam (Table 3). These similar effects observed for different studies indicate the reproducibility of PMR tests if experimental parameters are similar. The fact that the observed activity of embryos for the three chemicals was consistent with the expected activity assumed from the MOA suggests that PMR can be a valuable test to detect mode of action of neuroactive substances if steady state of concentrations can be achieved within exposure window and the respective receptor is available.

\section{Comparison of LMR with different light regimes Case study: Pentylenetetrazole (PTZ)}

There are two major types of LMR reported in the literature: The non-stimulus LMR either conducted in constant dark or light phase only and the LMR using alternating light-dark cycles (LMR-L/D). A comparative assessment of the different types of LMR was difficult due to the use of different endpoint parameters such as total distance moved, time spent on locomotion, swimming speed, mean turn angle, etc. PTZ was the only chemical for which sufficient data could be identified to enable a comparative assessment (Fig. 4). In the LMR-L/D, PTZ induces a behavior which is opposite to that observed in control embryos, i.e., high activity in dark period and low activity in light period for embryos in untreated solution [31, 115]. Therefore, all hyperactivity effects were recorded during the light phase in the LMR-L/D tests with PTZ. In the LMR, hyperactivity was recorded irrespective of the analysis being conducted in continuous light or dark phase. Interestingly, effect concentrations for hyperactivity reported for both setups in all the studies were within a variation factor of 10 . This shows that the hypoactivity observed only in the LMR-L/D tests was mainly driven by alternating light/dark cycles. This suggests that PTZ might react differentially under alternating light conditions and the LMR-L/D could be utilized as an extensive diagnostic tool for such epileptic effects in zebrafish.

\section{Conclusions}

Based on the assessment of published zebrafish embryo behavior studies it was possible to identify major factors impacting the magnitude and type of response in behavioral assays. Exposure duration, exposure concentration, endpoint parameter and developmental stage were the most influential parameters. Understanding and controlling these factors and potentially revising/harmonizing protocols would help reduce variability of results for hazard assessment of chemicals.

The review was motivated by the hypothesis that the MoA of chemicals may be reflected by the type of response in behavioral assays, i.e., whether hypo- or hyperactivity is induced by the exposure. The data indicated that a clear association of the response with the mode of action was difficult (e.g., 18 and 62\% consistency for organophosphates and anticonvulsants, respectively), partially also caused by experimental limitations and diversity of protocols used. Despite the low number of STC studies, the STC test appears to reveal the most consistent results with respect to the expected hyperactivity of a substance (especially for organophosphates). However, limited biotransformation capacity and uptake of chemicals into the embryos may affect the detectability and sensitivity of hyperactivity in the STC as was shown by the effects of the organophospate compounds, chlorpyrifos and diazinon. The PMR also shows great potential to predict neuroactive MoA; however, the use of single exposure concentrations in many PMR studies limited the appropriate evaluation of its possible potential. LMR $(\mathrm{L} / \mathrm{D})$ showed $64 \%$ of the expected hypoactivity related to the MoA of anticonvulsants. However, the anticipated hyperactivity for organophosphates could not be shown in most cases and which could be partially attributed to long exposure duration (e.g., 3-5 days). Long exposure durations may impact the neuronal development due to axonal defects or seizure-induced paralysis [111, 123]. Hence, compromising the function/structure of the nervous system and indirectly resulting in hypoactivity. The different LMR test methods may only acquire the ability to predict hyperactivity if the exposure duration is significantly reduced as shown in the PTZ studies and as reported in Leuthold et al. [68]. The possibility 
to discriminate neuro-developmental effects from direct functional effects may also improve MoA prediction. Finally, it is evident that behavioral tests are capable of screening neuroactive substances and a combination of the four tests considered in this review will be more powerful and reliable than the individual tests alone. Nonetheless, the full potential of these methods for risk assessment of chemicals cannot be realized until the impacts of experimental parameters are addressed more systematically in comparative studies.

\section{Recommendations}

The experimental design is always strongly related to the research question. Hence, the type of assays and conditions used for behavioral tests may be different depending on the goal of the study. The perspective in this manuscript was to analyze the comparability and reproducibility of behavioral test results for identifying neuroactivity MoA within a prospective and diagnostic risk assessment framework. The recommendations given below are based on this particular goal but may be different for other research questions. With respect to this focus and the results of this review, we suggest to particularly address the most important experimental parameters in behavioral assays:

1. Exposure duration could have a strong impact on behavioral outcomes for several reasons such as biotransformation rates or overall kinetics. From the data used in this review, it appears that functional neurotoxicity can be provoked already by relative short exposure periods, while developmental neurotoxicity is rather detected by long-term exposure exceeding $24 \mathrm{~h}$ and including early developmental stages. Hence, MoA-specific neuroactivity may rather be detected when using short-term exposure scenarios $(<24-32 \mathrm{~h}$ of exposure $)$ provided that uptake and biotransformation are not limiting the availability of the compound at the target site.

2. Behavioral tests are sensitive to exposure concentrations and responses may change within a range of low to high concentrations (e.g., due to seizure paralysis caused by high overstimulation or interfering of developmental toxicity at high exposure concentrations). Therefore, test design should include a range of concentrations that allow capturing of the potential transition from low dose hyperactivity to high dose hypoactivity effects. Concentration-response relationships should also be related to lethal effect concentrations at defined exposure periods and to predicted concentrations causing baseline toxicity. This will indicate whether behavioral effects may have been caused by unspecific secondary responses to overt toxicity.

3. Zebrafish embryos develop rapidly and their normal patterns of embryonic movements change with developmental stage. Hence, harmonization of protocols with regard to the developmental stages used for assessment is likely to increase reproducibility and reliability of results. Factors such as biotransformation requirement of the substance and availability of target receptor should be considered during experimental design.

4. For uniformity reasons, it seems from the analyzed literature that it is generally desirable to select distance moved for LMR and LMR-L/D and frequency of STC as optimal endpoint parameters. Other parameters could be used additionally until proof of usability.

5. Based on the review, we suggest to consider the following parameters to be used for the different behavioral assays.

\section{STC:}

- Exposure duration: 2-26 hpf $( \pm 2)$. Shorter durations maybe used for MoA analysis, for indirect assessment of toxicokinetics or to distinguish between acute and developmental effects;

- Selection of exposure concentration should relate to lethal or sublethal concentrations such as the $\mathrm{LC}_{50}$ at $24 \mathrm{hpf}$ as the highest test concentrations and a full concentration-response analysis should be performed;

- Endpoint parameter: Frequency or number of STC;

- Developmental stage: Measurement should be conducted between 23 and 25 hpf (based on the age at which maximum STC is observed) to account for the stage dependency of the frequency.

\section{PMR:}

- Exposure duration: 2-32 hpf $( \pm 2)$. Shorter durations within this period may be used for MoA analysis or indirect assessment of toxicokinetics or to distinguish between acute and developmental effects;

- Selection of exposure concentration should relate to lethal or sublethal concentrations such as the $\mathrm{LC}_{50}$ at 24 or $48 \mathrm{hpf}$ as the highest test concentrations and a full concentration-response analysis should be performed;

- Endpoint parameter: Movement activity as used in Kokel et al. [60] and Copmans et al. [21];

- Analysis duration: 30-s measurement from $30 \mathrm{hpf}$. 


\section{LMR or LMR-L/D:}

- Exposure duration: 2-120 hpf for developmental neurotoxicity assessment. Short durations, e.g., from 96 to $120 \mathrm{hpf}$ may be appropriate for identifying acute neuroactivity effects not related to neurodevelopmental toxicity;

- Selection of exposure concentration should relate to lethal or sublethal concentrations such as the $\mathrm{LC}_{50}$ at 72 or $96 \mathrm{hpf}$ as the highest test concentrations and a full concentration-response analysis should be performed;

- Endpoint parameter: Total distance moved and other endpoints such as swimming duration and velocity could be used as additional.

\section{Supplementary information}

Supplementary information accompanies this paper at https://doi. org/10.1186/s12302-019-0270-5.

Additional file 1. Detailed comparison of individual substances for all 18 substances represented in Figure 3 of main text. Table S1. Sources of fish embryo lethality data used in Figure 3 of main text. Figure S1. Summary of the literature selection process in KNIME.

Additional file 2. Excel sheet 1-111 Literature search hits and the corresponding chemicals. Excel sheet 2-31 chemical hits after regrouping and elimination of chemicals with less than 3 entries. Excel sheet 3 -Final 18 chemical hits and the experimental parameters analyzed in the main text of this study. Excel sheet 4-Summary of comparative analysis performed in this study as shown in Table 3 of the main text.

\section{Abbreviations}

MoA: mode of action; mins: minutes; AChE: acetylcholinesterase; nAChr: nicotine receptors; GABA: gamma-aminobutyric acid receptors; STC: spontaneous tail coiling; PMR: photomotor response; LMR: locomotor response; LMR-L/D: alternating light- and dark-induced locomotor response; DMSO: dimethyl sulfoxide; PTZ: pentylenetetrazole.

\section{Acknowledgements}

The critical comments and discussions of Wibke Busch, Rolf Altenburger and Nils Klüver to the manuscript are gratefully acknowledged. Parts of this study were presented at the SETAC EU conference in Rome 2018.

\section{Authors' contributions}

$\mathrm{AO}, \mathrm{SS}$ and EK conceptualized the study. All authors provided comments for the design of the study. AO performed the literature search, analyzed the results and drafted the manuscript. All authors edited and reviewed the manuscript. All authors read and approved the final manuscript.

\section{Funding}

This work was financially supported by the German bmbf project "Neurobox" (German Ministry of Education and Science, FKZ 02WRS1419F). We are also acknowledging financial support through the Helmholtz Gemeinschaft Deutscher Forschungszentren_HGF program topic "Chemicals in the Environment"_CITE.

\section{Availability of data and materials}

All data generated or analysed during this study are included in this published article [and its additional files].
Ethics approval and consent to participate

Not applicable.

\section{Consent for publication}

Not applicable.

\section{Competing interests}

The authors declare that they have no competing interests.

\section{Author details}

${ }^{1}$ Dept. Bioanalytical Ecotoxicology, Helmholtz Centre for Environmental Research-UFZ, Permoserstr. 15, 04318 Leipzig, Germany. ${ }^{2}$ Institute for Environmental Sciences, University of Koblenz-Landau, 76829, Fortstraße 7, Landau, Germany.

Received: 19 August 2019 Accepted: 19 October 2019

Published online: 09 November 2019

\section{References}

1. Afrikanova T, Serruys A-SK, Buenafe OEM et al (2013) Validation of the zebrafish pentylenetetrazol seizure model: locomotor versus electrographic responses to antiepileptic drugs. PLoS ONE 8:e54166. https:// doi.org/10.1371/journal.pone.0054166

2. Ali S, Champagne DL, Richardson MK (2012) Behavioral profiling of zebrafish embryos exposed to a panel of 60 water-soluble compounds. Behav Brain Res 228:272-283. https://doi.org/10.1016/j.bbr.2011.11.020

3. Bae M-J, Park Y-S (2014) Biological early warning system based on the responses of aquatic organisms to disturbances: a review. Sci Total Environ 466-467:635-649. https://doi.org/10.1016/j.scitotenv.2013.07.075

4. Bailey JM, Oliveri AN, Karbhari N et al (2016) Persistent behavioral effects following early life exposure to retinoic acid or valproic acid in zebrafish. Neurotoxicology 52:23-33. https://doi.org/10.1016/j.neuro .2015.10.001

5. Baraban SC, Taylor MR, Castro PA, Baier AH (2005) Pentylenetetrazole induced changes in zebrafish behavior, neural activity and c-fos expression. Neuroscience 131(3):759-768. https://doi.org/10.1016/j.neuro science.2004.11.031

6. Basnet R, Zizioli D, Taweedet S et al (2019) Zebrafish larvae as a behavioral model in neuropharmacology. Biomedicines 7:23. https://doi. org/10.3390/biomedicines7010023

7. Baxendale S, Holdsworth CJ, Meza Santoscoy PL et al (2012) Identification of compounds with anti-convulsant properties in a zebrafish model of epileptic seizures. Dis Model Mech 5:773-784. https://doi. org/10.1242/dmm.010090

8. Beekhuijzen M, de Koning C, Flores-Guillén ME et al (2015) From cutting edge to guideline: a first step in harmonization of the zebrafish embryotoxicity test (ZET) by describing the most optimal test conditions and morphology scoring system. Reprod Toxicol 56:64-76. https ://doi.org/10.1016/j.reprotox.2015.06.050

9. Beker van Woudenberg A, Snel C, Rijkmans E et al (2014) Zebrafish embryotoxicity test for developmental (neuro)toxicity: demo case of an integrated screening approach system using anti-epileptic drugs. Reprod Toxicol 49:101-116. https://doi.org/10.1016/j.repro tox.2014.07.082

10. Berghmans S, Hunt J, Roach A, Goldsmith P (2007) Zebrafish offer the potential for a primary screen to identify a wide variety of potential anticonvulsants. Epilepsy Res 75:18-28. https://doi.org/10.1016/j.eplep syres.2007.03.015

11. Brack W, Aissa SA, Backhaus T et al (2019) Effect-based methods are key. The European Collaborative Project SOLUTIONS recommends integrating effect-based methods for diagnosis and monitoring of water quality. Environ Sci Eur 31:10. https://doi.org/10.1186/s12302-019-0192-2

12. BraunbeckT, Böttcher $M$, Hollert $\mathrm{H}$ et al (2005) Towards an alternative for the acute fish LC50 test in chemical assessment: the fish embryo toxicity test goes multi-species-an update. ALTEX-Altern Anim Exp 22(2):87-102

13. Brox S, Ritter AP, Küster E, Reemtsma T (2014) A quantitative HPLC-MS/ MS method for studying internal concentrations and toxicokinetics of 
34 polar analytes in zebrafish (Danio rerio) embryos. Anal Bioanal Chem 406:4831-4840. https://doi.org/10.1007/s00216-014-7929-y

14. Bugel SM, Tanguay RL (2018) Multidimensional chemobehavior analysis of flavonoids and neuroactive compounds in zebrafish. Toxicol Appl Pharmacol 344:23-34. https://doi.org/10.1016/j.taap.2018.02.019

15. Busch W, Schmidt S, Kühne R et al (2016) Micropollutants in European rivers: a mode of action survey to support the development of effectbased tools for water monitoring. Environ Toxicol Chem 35:1887-1899. https://doi.org/10.1002/etc.3460

16. Cao F, Souders CL, Li P et al (2018) Biological impacts of organophosphates chlorpyrifos and diazinon on development, mitochondrial bioenergetics, and locomotor activity in zebrafish (Danio rerio). Neurotoxicol Teratol 70:18-27. https://doi.org/10.1016/j.ntt.2018.10.001

17. Casida JE, Durkin KA (2013) Neuroactive insecticides: targets, selectivity, resistance, and secondary effects. Annu Rev Entomol 58:99-117. https ://doi.org/10.1146/annurev-ento-120811-153645

18. Chambers J, Oppenheimer SF (2004) Organophosphates, serine esterase inhibition, and modeling of organophosphate toxicity. Toxicol Sci 77:185-187

19. Chen TH, Wang YH, Wu YH (2011) Developmental exposures to ethanol or dimethylsulfoxide at low concentrations alter locomotor activity in larval zebrafish: implications for behavioral toxicity bioassays. Aquat Toxicol 102:162-166. https://doi.org/10.1016/j.aquatox.2011.01.010

20. Colwill RM, Creton R (2011) Locomotor behaviors in zebrafish (Danio rerio) larvae. Behav Process 86:222-229. https://doi.org/10.1016/J. BEPROC.2010.12.003

21. Copmans D, Meinl T, Dietz C et al (2016) A KNIME-based analysis of the zebrafish photomotor response clusters the phenotypes of 14 classes of neuroactive molecules. J Biomol Screen 21:427-436. https://doi. org/10.1177/1087057115618348

22. Cowden J, Padnos B, Hunter D et al (2012) Developmental exposure to valproate and ethanol alters locomotor activity and retino-tectal projection area in zebrafish embryos. Reprod Toxicol 33:165-173. https ://doi.org/10.1016/J.REPROTOX.2011.11.111

23. Crofton K, Mundy W (2011) Developmental neurotoxicity testing: recommendations for developing alternative methods for the screening and prioritization of chemicals. Altex 28:9-15

24. Dach K, Yaghoobi B, Schmuck MR et al (2019) Teratological and behavioral screening of the national toxicology program 91-compound library in zebrafish (Danio rerio). Toxicol Sci 167:77-91. https://doi. org/10.1093/toxsci/kfy266

25. Dale K, Ellingsen S, Dale K et al (2017) Vitamin E reduces endosulfaninduced toxic effects on morphology and behavior in early development of zebrafish (Danio rerio). Food Chem Toxicol 101:84-93. https:// doi.org/10.1016/j.fct.2017.01.004

26. Damstra T (1978) Environmental chemicals and nervous system dysfunction. Yale J Biol Med 51:457-468

27. De Esch C, Van Der Linde H, Slieker R et al (2012) Locomotor activity assay in zebrafish larvae: influence of age, strain and ethanol. Neurotoxicol Teratol 34:425-433. https://doi.org/10.1016/j.ntt.2012.03.002

28. Diekmann M, Nagel R (2005) Different survival rates in zebrafish (Danio rerio) from different origins. J Appl Ichthyol 21:451-454. https://doi.org/ 10.1111/.1439-0426.2005.00622.x

29. Doke SK, Dhawale SC (2015) Alternatives to animal testing: a review. Saudi Pharm J 23:223-229. https://doi.org/10.1016/J.JSPS.2013.11.002

30. Ek F, Malo M, Andersson MÅ et al (2016) Behavioral analysis of dopaminergic activation in zebrafish and rats reveals similar phenotypes. ACS Chem Neurosci 7:54. https://doi.org/10.1021/acschemneuro.6b00014

31. Ellis LD, Seibert J, Soanes KH (2012) Distinct models of induced hyperactivity in zebrafish larvae. Brain Res 1449:46-59. https://doi. org/10.1016/j.brainres.2012.02.022

32. Ellis LD, Soanes KH (2012) A larval zebrafish model of bipolar disorder as a screening platform for neuro-therapeutics. Behav Brain Res 233:450-457. https://doi.org/10.1016/J.BBR.2012.05.043

33. Embry MR, Belanger SE, Braunbeck TA et al (2010) The fish embryo toxicity test as an animal alternative method in hazard and risk assessment and scientific research. Aquat Toxicol 97:79-87. https://doi. org/10.1016/j.aquatox.2009.12.008

34. Fetter E, Baldauf L, Da Fonte DF et al (2015) Comparative analysis of goitrogenic effects of phenylthiourea and methimazole in zebrafish embryos. Reprod Toxicol 57:10-20. https://doi.org/10.1016/j.repro tox.2015.04.012

35. Fischer FC, Cirpka OA, Goss K-U et al (2018) Application of experimental polystyrene partition constants and diffusion coefficients to predict the sorption of neutral organic chemicals to multiwell plates in in vivo and in vitro bioassays. Environ Sci Technol 52:13511-13522. https://doi. org/10.1021/acs.est.8b04246

36. Fischer FC, Henneberger L, König M et al (2017) Modeling exposure in the Tox21 in vitro bioassays. Chem Res Toxicol 30:1197-1208. https:// doi.org/10.1021/acs.chemrestox.7b00023

37. Fitzgerald JA, Kirla KT, Zinner CP, vom Berg CM (2019) Emergence of consistent intra-individual locomotor patterns during zebrafish development. Sci Rep 9:13647. https://doi.org/10.1038/s41598-019-49614-y

38. Fraser TWK, Khezri A, Jusdado JGH et al (2017) Toxicant induced behavioural aberrations in larval zebrafish are dependent on minor methodological alterations. Toxicol Lett 276:62-68. https://doi.org/10.1016/j.toxle t.2017.05.021

39. Gauthier PT, Vijayan MM (2018) Nonlinear mixed-modelling discriminates the effect of chemicals and their mixtures on zebrafish behavior. Sci Rep 8(1):1999. https://doi.org/10.1038/s41598-018-20112-x

40. Giacomini NJ, Rose B, Kobayashi K, Guo S (2006) Antipsychotics produce locomotor impairment in larval zebrafish. Neurotoxicol Teratol 28:245-250. https://doi.org/10.1016/j.ntt.2006.01.013

41. Glazer L, Wells CN, Drastal M et al (2017) Developmental exposure to low concentrations of two brominated flame retardants, BDE-47 and BDE-99, causes life-long behavioral alterations in zebrafish. Neurotoxicology 66:221-232. https://doi.org/10.1016/j.neuro.2017.09.007

42. Grunwald DJ, Kimmel CB, Westerfield M et al (1988) A neural degeneration mutation that spares primary neurons in the zebrafish. Dev Biol 126:115-128

43. Gülden M, Mörchel S, Seibert H (2001) Factors influencing nominal effective concentrations of chemical compounds in vitro: cell concentration. Toxicol In Vitro 15:233-243. https://doi.org/10.1016/S0887 -2333(01)00008-X

44. Gülden M, Seibert H (2005) Impact of bioavailability on the correlation between in vitro cytotoxic and in vivo acute fish toxic concentrations of chemicals. Aquat Toxicol 72:327-337. https://doi.org/10.1016/J.AQUAT OX.2005.02.002

45. Guryev V, Koudijs MJ, Berezikov E et al (2006) Genetic variation in the zebrafish. Genome Res 16:491. https://doi.org/10.1101/gr.4791006

46. Hamm JT, Ceger P, Allen D et al (2019) Characterizing sources of variability in zebrafish embryo screening protocols. Altex 36:103-120. https ://doi.org/10.14573/altex.1804162

47. Hill AJ, Teraoka H, Heideman W, Peterson RE (2005) Zebrafish as a mode vertebrate for investigating chemical toxicity. Toxicol Sci 86:6-19. https ://doi.org/10.1093/toxsci/kfi110

48. Horstick EJ, Bayleyen Y, Sinclair JL, Burgess HA (2017) Search strategy is regulated by somatostatin signaling and deep brain photoreceptors in zebrafish. BMC Biol 15:4. https://doi.org/10.1186/s12915-016-0346-2

49. Huang Y, Cartlidge R, Walpitagama M et al (2018) Unsuitable use of DMSO for assessing behavioral endpoints in aquatic model species. Sci Total Environ 615:107-114. https://doi.org/10.1016/j.scito tenv.2017.09.260

50. Ingebretson JJ, Masino MA (2013) Quantification of locomotor activity in larval zebrafish: considerations for the design of high-throughput behavioral studies. Front Neural Circuits 7:1-9. https://doi.org/10.3389/ fncir.2013.00109

51. Irons TD, Kelly P, Hunter DL et al (2013) Acute administration of dopaminergic drugs has differential effects on locomotion in larval zebrafish. Pharmacol Biochem Behav 103:792-813. https://doi.org/10.1016/j. pbb.2012.12.010

52. Irons TD, Macphail RC, Hunter DL, Padilla S (2010) Acute neuroactive drug exposures alter locomotor activity in larval zebrafish. Neurotoxicol Teratol 32:84-90. https://doi.org/10.1016/j.ntt.2009.04.066

53. Jarema KA, Hunter DL, Shaffer RM et al (2015) Acute and developmental behavioral effects of flame retardants and related chemicals in zebrafish. Neurotoxicol Teratol 52:194-209. https://doi.org/10.1016/j. ntt.2015.08.010

54. Jin Y, Liu Z, Peng T, Fu Z (2015) The toxicity of chlorpyrifos on the early life stage of zebrafish: asurvey on the endpoints at development, 
locomotor behavior, oxidative stress and immunotoxicity. Fish Shellfish Immunol 43:405-414. https://doi.org/10.1016/j.fsi.2015.01.010

55. Kais B, Schneider KE, Keiter S et al (2013) DMSO modifies the permeability of the zebrafish (Danio rerio) chorion-implications for the fish embryo test (FET). Aquat Toxicol 140-141:229-238. https://doi. org/10.1016/j.aquatox.2013.05.022

56. Kienle C, Köhler H-R, Gerhardt A (2009) Behavioural and developmental toxicity of chlorpyrifos and nickel chloride to zebrafish (Danio rerio) embryos and larvae. Ecotoxicol Environ Saf 72:1740-1747. https://doi. org/10.1016/J.ECOENV.2009.04.014

57. Kimmel CB, Ballard WW, Kimmel SR et al (1995) Stages of embryonic development of the zebrafish. Dev Dyn 203:253-310. https://doi. org/10.1002/aja.1002030302

58. Kimmel CB, Patterson J, Kimmel RO (1974) The development and behavioral characteristics of the startle response in the zebra fish. Dev Psychobiol 7:47-60. https://doi.org/10.1002/dev.420070109

59. Klüver $\mathrm{N}$, König M, Ortmann J et al (2015) The fish embryo toxicity test (FET) -identification of compounds with weak toxicity and analysis of behavioral effects to improve prediction of acute toxicity for neurotoxic compounds. Environ Sci Technol. https://doi.org/10.1021/acs.est.5b019 10

60. Kokel D, Bryan J, Laggner C et al (2010) Rapid behavior-based identification of neuroactive small molecules in the zebrafish. Nat Chem Biol 6:231-237. https://doi.org/10.1038/nchembio.307

61. Kristofco LA, Colon Cruz L, Haddad SP et al (2016) Age matters: developmental stage of Danio rerio larvae influences photomotor response thresholds to diazinion or diphenhydramine. Aquat Toxicol 170:344354. https://doi.org/10.1016/j.aquatox.2015.09.011

62. Küster E, Altenburger R (2007) Suborganismic and organismic effects of aldicarb and its metabolite aldicarb-sulfoxide to the zebrafish embryo (Danio rerio). Chemosphere 68:751-760. https://doi.org/10.1016/j. chemosphere.2006.12.093

63. Lange M, Neuzeret F, Fabreges B et al (2013) Inter-individual and interstrain variations in zebrafish locomotor ontogeny. PLOS ONE 8:e70172. https://doi.org/10.1371/journal.pone.0070172

64. Lawrence C (2007) The husbandry of zebrafish (Danio rerio): a review. Aquaculture 269:1-20. https://doi.org/10.1016/j.aquacultur e.2007.04.077

65. Lazic SE, Grote HE, Blakemore C et al (2006) Neurogenesis in the R6/1 transgenic mouse model of Huntington's disease: effects of environmental enrichment. Eur J Neurosci 23:1829-1838. https://doi.org/10.11 $11 / j .1460-9568.2006 .04715 . x$

66. Legradi J, el Abdellaoui N, van Pomeren M, Legler J (2015) Comparability of behavioural assays using zebrafish larvae to assess neurotoxicity. Environ Sci Pollut Res Int 22:16277-16289. https://doi.org/10.1007/ s1 1356-014-3805-8

67. Legradi JB, Di Paolo C, Kraak MHS et al (2018) An ecotoxicological view on neurotoxicity assessment. Environ Sci Eur 30:46. https://doi. org/10.1186/s12302-018-0173-x

68. Leuthold D, Klü N, Altenburger R, Busch W (2019) Can environmentally relevant neuroactive chemicals specifically be detected with the locomotor response test in zebrafish embryos? Environ Sci Technol 53(1):482-493. https://doi.org/10.1021/acsest 8b04327

69. Levin ED, Swain HA, Donerly S, Linney E (2004) Developmental chlorpyrifos effects on hatchling zebrafish swimming behavior. Neurotoxicol Teratol 26(6):719-723. https://doi.org/10.1016/j.ntt.2004.06.013

70. Li F, Lin J, Liu X et al (2018) Characterization of the locomotor activities of zebrafish larvae under the influence of various neuroactive drugs. Ann Transl Med. https://doi.org/10.21037/atm.2018.04.25

71. Li R, Zhang L, Shi Q et al (2018) A protective role of autophagy in TDCIPP-induced developmental neurotoxicity in zebrafish larvae. Aquat Toxicol 199:46-54. https://doi.org/10.1016/j.aquatox.2018.03.016

72. Lillicrap A, Belanger S, Burden N et al (2016) Alternative approaches to vertebrate ecotoxicity tests in the 21st century: a review of developments over the last 2 decades and current status. Environ Toxicol Chem 35:2637-2646. https://doi.org/10.1002/etc.3603

73. Liu Y, Carmer R, Zhang G et al (2015) Statistical analysis of zebrafish locomotor response. PLoS ONE 10:e0139521. https://doi.org/10.1371/ journal.pone.0139521
74. Long SM, Liang FY, Wu Q et al (2014) Identification of marine neuroactive molecules in behaviour-based screens in the larval zebrafish. Mar Drugs 12:3307-3322. https://doi.org/10.3390/md12063307

75. Loucks E, Carvan MJ (2004) Strain-dependent effects of developmental ethanol exposure in zebrafish. Neurotoxicol Teratol 26:745-755. https:// doi.org/10.1016/J.NTT.2004.06.017

76. MacPhail RC, Brooks J, Hunter DL et al (2008) Locomotion in larval zebrafish: influence of time of day, lighting and ethanol. Neurotoxicology 30:52-58. https://doi.org/10.1016/j.neuro.2008.09.011

77. Martinez CS, Feas DA, Siri M et al (2018) In vivo study of teratogenic and anticonvulsant effects of antiepileptics drugs in zebrafish embryo and larvae. Neurotoxicol Teratol 66:17-24. https://doi.org/10.1016/j. ntt.2018.01.008

78. Massei R, Vogs C, Renner P et al (2015) Differential sensitivity in embryonic stages of the zebrafish (Danio rerio): the role of toxicokinetics for stage-specific susceptibility for azinphos-methyl lethal effects. Aquat Toxicol 166:36-41. https://doi.org/10.1016/j.aquatox.2015.06.011

79. Mora-Zamorano FX, Svoboda KR, Carvan MJ (2016) The nicotineevoked locomotor response: a behavioral paradigm for toxicity screening in zebrafish (Danio rerio) embryos and eleutheroembryos exposed to methylmercury. PLoS ONE 11:e0154570. https://doi.org/10.1371/ journal.pone. 0154570

80. Oliveri AN, Bailey JM, Levin ED (2015) Developmental exposure to organophosphate flame retardants causes behavioral effects in larval and adult zebrafish HHS Public Access. Neurotoxicol Teratol 52:220-227. https://doi.org/10.1016/j.ntt.2015.08.008

81. Oliveri AN, Levin ED (2019) Dopamine D 1 and D 2 receptor antagonism during development alters later behavior in zebrafish. Behav Brain Res 356:250-256. https://doi.org/10.1016/j.bbr.2018.08.028

82. Padilla S, Hunter DL, Padnos B et al (2011) Assessing locomotor activity in larval zebrafish: influence of extrinsic and intrinsic variables. Neurotoxicol Teratol 33:624-630. https://doi.org/10.1016/j.ntt.2011.08.005

83. Parker MO, Millington ME, Combe FJ, Brennan CH (2012) Housing conditions differentially affect physiological and behavioural stress responses of zebrafish, as well as the response to anxiolytics. PLOS ONE 7:e34992. https://doi.org/10.1371/journal.pone.0034992

84. Pelka KE, Henn K, Keck A et al (2017) Size does matter—determination of the critical molecular size for the uptake of chemicals across the chorion of zebrafish (Danio rerio) embryos. Aquat Toxicol 185:1-10. https:// doi.org/10.1016/j.aquatox.2016.12.015

85. Peng X, Lin J, Zhu Y et al (2016) Anxiety-related behavioral responses of pentylenetetrazole-treated zebrafish larvae to light-dark transitions. Pharmacol Biochem Behav 145:55-65. https://doi.org/10.1016/j. pbb.2016.03.010

86. Raftery TD, Isales GM, Yozzo KL, Volz DC (2014) High-content screening assay for identification of chemicals impacting spontaneous activity in zebrafish embryos. Environ Sci Technol 48:804-810. https://doi. org/10.1021/es404322p

87. Raftery TD, Volz DC (2015) Abamectin induces rapid and reversible hypoactivity within early zebrafish embryos. Neurotoxicol Teratol 49:10-18. https://doi.org/10.1016/j.ntt.2015.02.006

88. Ramsay JM, Feist GW, Varga ZM et al (2006) Whole-body cortisol is an indicator of crowding stress in adult zebrafish, Danio rerio. Aquaculture 258:565-574. https://doi.org/10.1016/J.AQUACULTURE.2006.04.020

89. Reif DM, Truong L, Mandrell D et al (2016) High-throughput characterization of chemical-associated embryonic behavioral changes predicts teratogenic outcomes. Arch Toxicol 90:1459-1470. https://doi. org/10.1007/s00204-015-1554-1

90. Reuter I, Knaup S, Romanos M et al (2016) Developmental exposure to acetaminophen does not induce hyperactivity in zebrafish larvae. J Neural Transm 123(8):841-848

91. Ribas L, Valdivieso A, Díaz N, Piferrer F (2017) Appropriate rearing density in domesticated zebrafish to avoid masculinization: links with the stress response. J Exp Biol 220:1056-1064. https://doi.org/10.1242/ jeb.144980

92. Richendrfer H, Creton R, Colwill R (2014) The embryonic zebrafish as a model system to study the effects of environmental toxicants on behavior. Zebrafish Nova Science Publishers, New York, pp 245-264

93. Richendrfer H, Pelkowski SD, Colwill RM, Créton R (2012) Developmental sub-chronic exposure to chlorpyrifos reduces anxiety-related 
behavior in zebrafish larvae. Neurotoxicol Teratol 34:458-465. https:// doi.org/10.1016/j.ntt.2012.04.010

94. Rico EP, Rosemberg DB, Seibt KJ et al (2011) Zebrafish neurotransmitter systems as potential pharmacological and toxicological targets. Neurotoxicol Teratol 33:608-617. https://doi.org/10.1016/..ntt.2011.07.007

95. Rombough P (2002) Role of gills in developing zebrafish. J Exp Biol 205:1787-1794

96. Russell WMS, Burch RL (1959) The principles of humane experimental technique, Methuen, London. ISBN 0900767782.https://altweb.jhsph .edu/pubs/books/humane_exp/het-toc. Accessed 28 Oct 2019

97. Russom CL, Lalone CA, Villeneuve DL, Ankley GT (2014) Development of an adverse outcome pathway for acetylcholinesterase inhibition leading to acute mortality. Environ Toxicol Chem 33:2157-2169. https://doi. org/10.1002/etc.2662

98. Saint-Amant L, Drapeau P (1998) Time course of the development of motor behaviors in the zebrafish embryo. J Neurobiol 37:622-632. https ://doi.org/10.1002/(SICI)1097-4695(199812)37:4\%3c622:AID-NEU10 \%3e3.0.CO;2-S

99. Scheil V, Kienle C, Osterauer R et al (2009) Effects of 3,4-dichloroaniline and diazinon on different biological organisation levels of zebrafish (Danio rerio) embryos and larvae. Ecotoxicology 18:355-363. https://doi. org/10.1007/s10646-008-0291-0

100. Schirmer K (2006) Proposal to improve vertebrate cell cultures to establish them as substitutes for the regulatory testing of chemicals and effluents using fish. Toxicology 224:163-183. https://doi.org/10.1016/j. tox.2006.04.042

101. Schmitt C, McManus M, Kumar N et al (2019) Comparative analyses of the neurobehavioral, molecular, and enzymatic effects of organophosphates on embryo-larval zebrafish (Danio rerio). Neurotoxicol Teratol 73:67-75. https://doi.org/10.1016/j.ntt.2019.04.002

102. Scholz S, Fischer S, Gündel U et al (2008) The zebrafish embryo model in environmental risk assessment - applications beyond acute toxicity testing. Environ Sci Pollut Res 15:394-404. https://doi.org/10.1007/ s11356-008-0018-z

103. Scholz S, Sela E, Blaha L et al (2013) A European perspective on alternatives to animal testing for environmental hazard identification and risk assessment. Regul Toxicol Pharmacol 67:506-530. https://doi. org/10.1016/j.yrtph.2013.10.003

104. Schreiber R, Altenburger R, Paschke A, Küster E (2008) How to deal with lipophilic and volatile organic substances in microtiter plate assays. Environ Toxicol Chem 27:1676. https://doi.org/10.1897/07-504.1

105. Selderslaghs IWT, Hooyberghs J, Blust R, Witters HE (2013) Assessment of the developmental neurotoxicity of compounds by measuring locomotor activity in zebrafish embryos and larvae. Neurotoxicol Teratol 37:44-56. https://doi.org/10.1016/j.ntt.2013.01.003

106. Selderslaghs IWT, Hooyberghs J, De Coen W, Witters HE (2010) Locomotor activity in zebrafish embryos: a new method to assess developmental neurotoxicity. Neurotoxicol Teratol 32:460-471. https://doi. org/10.1016/j.ntt.2010.03.002

107. Söderpalm B (2002) Anticonvulsants: aspects of their mechanisms of action. Eur J Pain 6:3-9. https://doi.org/10.1053/eujp.2001.0315

108. Squires RF, Saederup E, Crawley JN et al (1984) Convulsant potencies of tetrazoles are highly correlated with actions on GABA/benzodiazepine/ picrotoxin receptor complexes in brain. Life Sci 35:1439-1444. https:// doi.org/10.1016/0024-3205(84)90159-0

109. Steele WB, Kristofco LA, Corrales J et al (2018) Comparative behavioral toxicology with two common larval fish models: exploring relationships among modes of action and locomotor responses. Sci Total Environ 640-641:1587-1600. https://doi.org/10.1016/j.scitotenv.2018.05.402

110. Steenbergen PJ, Richardson MK, Champagne DL (2011) Patterns of avoidance behaviours in the light/dark preference test in young juvenile zebrafish: a pharmacological study. Behav Brain Res 222:15-25. https://doi.org/10.1016/j.bbr.2011.03.025

111. Stehr CM, Linbo TL, Incardona JP, Scholz NL (2006) The developmental neurotoxicity of fipronil: notochord degeneration and locomotor defects in zebrafish embryos and larvae. Toxicol Sci 92:270-278. https:// doi.org/10.1093/toxsci/kfj185

112. Sun L, Xu W, Peng T et al (2016) Developmental exposure of zebrafish larvae to organophosphate flame retardants causes neurotoxicity. Neurotoxicol Teratol 55:16-22. https://doi.org/10.1016/.ntt.2016.03.003
113. Thomas LT, Welsh L, Galvez F, Svoboda KR (2009) Acute nicotine exposure and modulation of a spinal motor circuit in embryonic zebrafish. Toxicol Appl Pharmacol 239:1-12. https://doi.org/10.1016/j. taap.2008.08.023

114. Tierney KB (2011) Behavioural assessments of neurotoxic effects and neurodegeneration in zebrafish. BBA Mol Basis Dis 1812:381-389. https ://doi.org/10.1016/j.bbadis.2010.10.011

115. Torres-Hernández BA, Colón LR, Rosa-Falero C et al (2016) Reversal of pentylenetetrazole-altered swimming and neural activity-regulated gene expression in zebrafish larvae by valproic acid and valerian extract. Psychopharmacology 233:2533-2547. https://doi.org/10.1007/ s00213-016-4304-z

116. Tufi S, Leonards P, Lamoree M et al (2016) Changes in neurotransmitter profiles during early zebrafish (Danio rerio) development and after pesticide exposure. Environ Sci Technol 50:3222-3230. https://doi. org/10.1021/acs.est.5b05665

117. Velki M, Di Paolo C, Nelles J et al (2017) Diuron and diazinon alter the behavior of zebrafish embryos and larvae in the absence of acute toxicity. Chemosphere 180:65-76. https://doi.org/10.1016/j.chemospher e.2017.04.017

118. Vliet SM, Ho TC, Volz DC (2017) Behavioral screening of the LOPAC 1280 library in zebrafish embryos. Toxicol Appl Pharmacol 329:241-248. https ://doi.org/10.1016/j.taap.2017.06.011

119. Wang Y, Chen J, Du C et al (2014) Characterization of retinoic acidinduced neurobehavioral effects in developing zebrafish. Environ Toxicol Chem 33:431-437. https://doi.org/10.1002/etc.2453

120. Watson FL, Schmidt H, Turman ZK et al (2014) Organophosphate pesticides induce morphological abnormalities and decrease locomotor activity and heart rate in Danio rerio and Xenopus laevis. Environ Toxicol Chem 33:1337-1345. https://doi.org/10.1002/etc.2559

121. Weichert FG, Floeter C, Artmann ASM, Kammann U (2017) Assessing the ecotoxicity of potentially neurotoxic substances - evaluation of a behavioural parameter in the embryogenesis of Danio rerio. Chemosphere 186:43-50. https://doi.org/10.1016/j.chemosphere.2017.07.136

122. Xia L, Zheng L, Zhou JL (2017) Effects of ibuprofen, diclofenac and paracetamol on hatch and motor behavior in developing zebrafish (Danio rerio). Chemosphere 182:416-425. https://doi.org/10.1016/J.CHEMO SPHERE.2017.05.054

123. Yang D, Lauridsen $H$, Buels K et al (2011) Chlorpyrifos-oxon disrupts zebrafish axonal growth and motor behavior. Toxicol Sci 121:146-159. https://doi.org/10.1093/toxsci/kfr028

124. Yang X, Lin J, Peng X et al (2017) Effects of picrotoxin on zebrafish larvae behaviors: a comparison study with PTZ. Epilepsy Behav 70:224-231. https://doi.org/10.1016/j.yebeh.2017.03.023

125. Yen J, Donerly S, Levin ED, Linney EA (2011) Differential acetylcholinesterase inhibition of chlorpyrifos, diazinon and parathion in larval zebrafish. Neurotoxicol Teratol 33:735-741. https://doi.org/10.1016/j. ntt.2011.10.004

126. Yozzo KL, Mcgee SP, Volz DC (2013) Adverse outcome pathways during zebrafish embryogenesis: a case study with paraoxon. Aquat Toxicol 126:346-354. https://doi.org/10.1016/j.aquatox.2012.09.008

127. Zellner D, Padnos B, Hunter DL et al (2011) Rearing conditions differentially affect the locomotor behavior of larval zebrafish, but not their response to valproate-induced developmental neurotoxicity. Neurotoxicol Teratol 33:674-679. https://doi.org/10.1016/j.ntt.2011.06.007

128. Zimmermann FF, Gaspary KV, Leite CE et al (2015) Embryological exposure to valproic acid induces social interaction deficits in zebrafish (Danio rerio): a developmental behavior analysis. Neurotoxicol Teratol 52:36-41. https://doi.org/10.1016/j.ntt.2015.10.002

129. Zindler F, Beedgen F, Braunbeck T (2019) Time-course of coiling activity in zebrafish (Danio rerio) embryos exposed to ethanol as an endpoint for developmental neurotoxicity (DNT) —hidden potential and underestimated challenges. Chemosphere 235:12-20. https://doi. org/10.1016/j.chemosphere.2019.06.154

\section{Publisher's Note}

Springer Nature remains neutral with regard to jurisdictional claims in published maps and institutional affiliations. 